\title{
Holomorphic curvature of Finsler metrics and complex geodesics
}

\author{
by Marco Abate and Giorgio Patrizio
}

\section{Introduction}

If $D$ is a bounded convex domain in $\mathbb{C}^{n}$, then the work of Lempert [L] and Royden-Wong [RW] (see also $[\mathrm{A}]$ ) show that given any point $p \in D$ and any non-zero tangent vector $v \in \mathbb{C}^{n}$ at $p$, there exists a holomorphic map $\varphi: U \rightarrow D$ from the unit disk $U \subset \mathbb{C}$ into $D$ passing through $p$ and tangent to $v$ in $p$ which is an isometry with respect to the hyperbolic distance of $U$ and the Kobayashi distance of $D$. Furthermore if $D$ is smooth and strongly convex then given $p$ and $v$ this holomorphic disk is uniquely determined.

For a general complex manifold it is hard to determine whether or not such complex curves, called complex geodesics in [V], exist. Therefore it is natural to investigate the special properties enjoyed by the Kobayashi metric of a strongly convex domain. In this case it is known that the Kobayashi metric is a strongly pseudoconvex smooth complex Finsler metric. Furthermore, for a suitable notion of holomorphic curvature (see below), this metric in convex domain has negative constant holomorphic curvature (cf. [W], [S], $[\mathrm{R}])$.

In $[\mathrm{AP}]$ it was started a systematic differential geometrical study of complex geodesics in the framework of complex Finsler metrics. As in ordinary Riemannian geometry it is natural to study geodesics as solutions of an extremal problem and not as globally lengthminimizing curves, so in our case the natural notion turns out to be the one of geodesic complex curves, i.e., of holomorphic maps from the unit disk into the manifold sending geodesics for the hyperbolic metric into geodesics for the given Finsler metric. For instance, the annulus in $\mathbb{C}$ has no complex geodesics in the sense of $[\mathrm{V}]$, whereas the usual universal covering map is a geodesic complex curve in the previous sense.

It was shown in $[\mathrm{AP}]$ that geodesic complex curves for complex Finsler metrics satisfy a system of partial differential equations and, under suitable hypotheses, it was given an uniqueness theorem. Here we shall be concerned with the question of existence.

The further ingredient needed to attack this problem is the notion of holomorphic curvature of complex Finsler metrics. Given a complex manifold $M$ and a complex Finsler metric $F: T^{1,0} M \rightarrow \mathbb{R}$, i.e., a nonnegative upper semicontinuous function such that

$$
F(p ; \lambda v)=|\lambda| F(p ; v)
$$

for all $(p ; v) \in T^{1,0} M$ and $\lambda \in \mathbb{C}$, the holomorphic curvature of $F$ at $p$ in the direction $v$ is the supremum of the Gaussian curvature at the origin of the (pseudo)hermitian metrics on the unit disk obtained by pulling back $F$ via holomorphic maps $\varphi: U \rightarrow M$ with $\varphi(0)=p$ and $\varphi^{\prime}(0)=\lambda v$ for some $\lambda \in \mathbb{C}^{*}$. Here, following $[\mathrm{H}]$, we make the choice of computing the Gaussian curvature using the weak laplacian rather then using Ahlfors' notion of supporting metrics; this approach seems to be more natural for our applications and has a better connection with the usual hermitian geometry. We remark that if $F$ is the norm associated to a hermitian metric, then $\mathrm{Wu}$ in $[\mathrm{Wu}]$ showed that this definition yields the 
usual holomorphic sectional curvature in the direction $v$. We shall see in section 2 that for smooth strongly pseudoconvex (see below for definitions) Finsler metrics this definition recovers the holomorphic sectional curvature defined by Kobayashi in [K2].

In section 1 we give a survey of the elementary implications of this notion of holomorphic curvature of complex Finsler metrics gotten by application of Ahlfors' lemma and of its sharp form due to Heins $[\mathrm{H}]$. For instance, as one could expect, it is easy to prove a hyperbolicity criterion in terms of negatively curved upper semicontinuous Finsler metric (cf. Corollary 1.5). As for the investigation of geodesic complex curves and differential geometric properties of intrinsic metrics, not much can be achieved without some smoothness assumptions. One of the few general facts obtained here is the explanation (Proposition 1.6) in terms of the holomorphic curvature of the known property of the Carathéodory metric that a holomorphic disk which is an isometry at one point is in fact an infinitesimal isometry at every point (i.e., an infinitesimal complex geodesic in the terminology introduced in [V]). Furthermore, in Proposition 1.7 it is given a very weak characterization of the Kobayashi metric which nevertheless is useful later in the paper.

In order to prove more significant results it is necessary to consider the case of smooth Finsler metric, i.e., such that $F \in C^{\infty}\left(T^{1,0} M \backslash\{\right.$ Zero section $\left.\}\right)$, which are in addition strongly pseudoconvex, that is such that for every $p \in M$ the indicatrix

$$
I_{p}(M)=\left\{v \in T^{1,0} M \mid F(p ; v)<1\right\}
$$

is strongly pseudoconvex. Under these assumptions in section 2 we show how to compute the holomorphic curvature $K_{F}$ of $F$ by means of a tensor explicitly defined in terms of $F$ and which agrees with the usual one in case $F$ is the norm associated to a hermitian metric. The expression we get has also been considered from a slightly different point of view by Royden [R] and Kobayashi [K2].

After this preparation we address the problem of the existence of geodesic complex curves in section 3 . Let

$$
A(\zeta)=\frac{-2 \bar{\zeta}}{1-|\zeta|^{2}}
$$

for $\zeta \in U$, and

$$
\Gamma_{; i}^{\alpha}=G^{\alpha \bar{\mu}} G_{\bar{\mu} ; i}
$$

where $G=F^{2}$, lower indices indicate derivatives (with respect to the coordinates of the manifold those after semicolon, with respect to the coordinates of the tangent space the others), $\left(G^{\alpha \bar{\beta}}\right)=\left(G_{\alpha \bar{\beta}}\right)^{-1}$ and we are using the usual summation convention. From results of $[\mathrm{AP}]$ it follows that the geodesic complex curves are holomorphic maps $\varphi: U \rightarrow M$ satisfying, for $\alpha=1, \ldots, n$,

$$
\left(\varphi^{\prime \prime}\right)^{\alpha}+A \cdot\left(\varphi^{\prime}\right)^{\alpha}+\Gamma_{; i}^{\alpha}\left(\varphi ; \varphi^{\prime}\right) \varphi^{i}=0,
$$

and an additional set of equations which automatically hold if along the curve the metric $F$ satisfies a Kähler condition introduced by Rund [Ru2], which reduces to the usual one for hermitian metrics.

The holomorphic solutions of (0.1) have nice properties on their own. For instance, they realize the holomorphic curvature at every point for the direction tangent to the 
disk, and if they are isometry at the origin then they are infinitesimal complex geodesics (cf. Proposition 3.2). Our first main result (Theorem 3.3) describes necessary and sufficient conditions for the holomorphic solvability of (0.1), and hence for the existence (and uniqueness) of complex geodesics through a given point and direction.

The characterization given in Theorem 3.3 is completely expressed in terms of the metric, but rather technical; to give a clearer geometric characterization it is necessary to bring the curvature into the picture. The previous list of properties of holomorphic solutions of (0.1) shows that a natural necessary condition is that $F$ has constant negative holomorphic curvature. This is almost sufficient; to get the correct geometric conditions it is necessary to introduce a further tensor, defined on the sphere bundle $S^{1,0} M=\left\{(p ; \xi) \in T^{1,0} M \mid F(p ; \xi)=1\right\}$. Set for $\alpha=1, \ldots, n$,

$$
H_{\alpha}(v)=H_{\alpha i \bar{\mu} \bar{\jmath}} \overline{v^{\mu}} v^{i} \overline{v^{j}}=\left[\left(G_{\tau \bar{\mu}} \Gamma_{; \alpha \bar{\jmath}}^{\tau}\right)_{i}-\left(G_{\tau \bar{\mu}} \Gamma_{; i \bar{\jmath}}^{\tau}\right)_{\alpha}\right] \overline{v^{\mu}} v^{i} \overline{v^{j}},
$$

where $\Gamma_{; i \bar{\jmath}}^{\alpha}=\left(G^{\alpha \bar{\mu}} G_{\bar{\mu} ; i}\right)_{; \bar{\jmath}}$. To understand the meaning of this tensor, let us consider the case of a hermitian metric. Then $H_{\alpha i \bar{\mu} \bar{\jmath}}=R_{i \alpha \bar{\mu} \bar{\jmath}}-R_{\alpha i \bar{\mu} \bar{\jmath}}$, where $R$ is the Riemannian curvature tensor of the Chern connection associated to the hermitian metric. In particular, $H_{\alpha i \bar{\mu} \bar{\jmath}} \equiv 0$ is equivalent to $\bar{\partial} T \equiv 0$, where $T$ is the torsion form of the Chern connection; $T$ is a $T^{1,0} M$-valued 2 -form vanishing exactly when the given metric is Kähler. In conclusion, $H$ may be interpreted as a torsion of the curvature; in fact, it can be shown that $H_{\alpha} \equiv 0$ is a simmetry condition - formally identical to (3.29) — on the curvature tensor of the Chern connection induced by the Finsler metric on the vertical subbundle of the two-fold tangent bundle $T^{1,0}\left(T^{1,0} M\right)$. We intend to pursue these matters elsewhere.

Using the tensor $H$ we may finally summarize our main results. We have (Theorem 3.6):

Theorem 0.1: Let $M$ be a complex manifold equipped with a strongly pseudoconvex smooth complete Finsler metric $F$. Then there exists a unique holomorphic solution $\varphi: U \rightarrow M$ of (0.1) with $\varphi(0)=p$ and $\varphi^{\prime}(0)=\xi$ for any $(p ; \xi) \in S^{1,0} M$ iff the holomorphic curvature $K_{F} \equiv-4$ and $H_{\alpha} \equiv 0$ for all $\alpha$.

In other words, the existence and uniqueness of holomorphic solutions of (0.1) is equivalent to constant negative holomorphic curvature and a simmetry condition on a curvature tensor. Furthermore, it turns out the the Kähler condition along a holomorphic solution of (0.1) holds iff it holds at one point, and thus (Corollary 3.9)

Theorem 0.2: Let $M$ be a complex manifold equipped with a strongly pseudoconvex smooth complete Finsler metric $F$. Assume that the holomorphic curvature $K_{F} \equiv-4$ and that $H_{\alpha} \equiv 0$ for all $\alpha$. Take $\left(p_{0} ; \xi_{0}\right) \in S^{1,0} M$. Then there is a (a fortiori unique) geodesic complex curve passing through $p_{0}$ tangent to $\xi_{0}$ iff the Kähler condition holds at $\left(p_{0} ; \xi_{0}\right)$.

From this result it is also possible to obtain a geometric characterization of the Kobayashi metric. The following corollary (Corollary 3.10) improves results of Pang [P] and it is closely related to those of Faran $[\mathrm{F}]$ :

Corollary 0.3: Let $F$ be a strongly pseudoconvex smooth complete Finsler metric with constant holomorphic curvature $K_{F} \equiv-4$ and such that $H_{\alpha} \equiv 0$ for all $\alpha$. Then $F$ is the Kobayashi metric of $M$. 
The second named author thanks the Max-Planck-Institut für Mathematik of Bonn for its hospitality and support while this paper was completed.

\section{Holomorphic curvature for semicontinuous metrics}

Let $U$ denote the unit disk in the complex plane. A pseudohermitian metric $\mu_{g}$ of scale $g$ on $U$ is the upper semicontinuous pseudometric on the tangent bundle of $U$ defined by

$$
\mu_{g}=g d \zeta \otimes d \bar{\zeta}
$$

where $g: U \rightarrow \mathbb{R}^{+}$is a non-negative upper semicontinuous function such that $S_{g}=g^{-1}(0)$ is a discrete subset of $U$.

If $\mu_{g}$ is a standard hermitian metric on $U$, i.e., $g$ is a $C^{2}$ positive function, the Gaussian curvature of $\mu_{g}$ is defined by

$$
K\left(\mu_{g}\right)=-\frac{1}{2 g} \Delta \log g
$$

where $\Delta$ denotes the usual Laplacian

$$
\Delta u=4 \frac{\partial^{2} u}{\partial \zeta \partial \bar{\zeta}}
$$

The (lower) generalized Laplacian of an upper semicontinuous function $u$ is defined by

$$
\Delta u(\zeta)=4 \liminf _{r \rightarrow 0} \frac{1}{r^{2}}\left\{\frac{1}{2 \pi} \int_{0}^{2 \pi} u\left(\zeta+r e^{i \theta}\right) d \theta-u(\zeta)\right\}
$$

It is worthwhile to remark explicitely some features of this definition. First of all, when $u$ is a function of class $C^{2}$ in a neighbourhood of the point $\zeta_{0},(1.4)$ actually reduces to (1.3). In fact, for $r$ small enough we can write

$$
\begin{aligned}
u\left(\zeta_{0}+r e^{i \theta}\right)=u\left(\zeta_{0}\right) & +\frac{\partial u}{\partial \zeta}\left(\zeta_{0}\right) r e^{i \theta}+\frac{\partial u}{\partial \bar{\zeta}}\left(\zeta_{0}\right) r e^{-i \theta} \\
& +\frac{1}{2} \frac{\partial^{2} u}{\partial \zeta^{2}}\left(\zeta_{0}\right) r^{2} e^{2 i \theta}+\frac{\partial^{2} u}{\partial \zeta \partial \bar{\zeta}}\left(\zeta_{0}\right) r^{2}+\frac{1}{2} \frac{\partial^{2} u}{\partial \bar{\zeta}^{2}}\left(\zeta_{0}\right) r^{2} e^{-2 i \theta}+o\left(r^{2}\right)
\end{aligned}
$$

hence

$$
\frac{1}{2 \pi} \int_{0}^{2 \pi} u\left(\zeta_{0}+r e^{i \theta}\right) d \theta-u(\zeta)=r^{2} \frac{\partial^{2} u}{\partial \zeta \partial \bar{\zeta}}\left(\zeta_{0}\right)+o\left(r^{2}\right),
$$

and the claim follows.

Second, if $u$ is an upper semicontinuous function, it is easy to see that $\Delta u \geq 0$ is equivalent to the submean property; so $\Delta u \geq 0$ iff $u$ is subharmonic.

Finally, if $\zeta_{0}$ is a point of local maximum for $u$, then clearly $\Delta u\left(\zeta_{0}\right) \leq 0$.

Now let $\mu_{g}$ be a pseudohermitian metric on $U$. Then the Gaussian curvature $K\left(\mu_{g}\right)$ of $\mu_{g}$ is the function defined on $U \backslash S_{g}$ by (1.2) — using the generalized Laplacian (1.4); 
clearly, if $\mu_{g}$ is a standard hermitian metric, $K\left(\mu_{g}\right)$ reduced to the usual Gaussian curvature.

The idea behind the classical Ahlfors lemma is to compare a generic pseudohermitian metric with an extremal one — usually the Poincaré metric. For $a>0$, let $g_{a}: U \rightarrow \mathbb{R}^{+}$ be defined by

$$
g_{a}(\zeta)=\frac{1}{a\left(1-|\zeta|^{2}\right)^{2}}
$$

then $\mu_{a}=g_{a} d \zeta \otimes d \bar{\zeta}$ is a hermitian metric of constant Gaussian curvature $K\left(\mu_{a}\right)=-4 a$. Of course, $\mu_{1}$ is the standard Poincaré metric on $U$.

The classical Ahlfors lemma is true in this more general situation:

Proposition 1.1: Let $\mu_{g}=g d \zeta \otimes d \bar{\zeta}$ be a pseudohermitian metric on $U$ such that $K\left(\mu_{g}\right) \leq-4 a$ on $U \backslash S_{g}$ for some $a>0$. Then $g \leq g_{a}$.

Proof: The proof follows closely the classical one due to Ahlfors. For the sake of completeness we report it here.

For $0<r<1$, define $U_{r}=\{\zeta \in \mathbb{C}|| \zeta \mid<r\}$ and $g_{a}^{r}: U_{r} \rightarrow \mathbb{R}^{+}$by

$$
g_{a}^{r}(\zeta)=\frac{1}{a\left(1-|\zeta|^{2} / r^{2}\right)^{2}}=g_{a}(\zeta / r)
$$

and set $f_{r}=g / g_{a}^{r}: U_{r} \rightarrow \mathbb{R}^{+}$. Being upper semicontinuous, $g$ is bounded on $\overline{U_{r}}$; since $g_{a}^{r}(\zeta) \rightarrow+\infty$ as $|\zeta| \rightarrow r$, there is a point $\zeta_{0} \in U_{r}$ of maximum for $f_{r}$. Clearly, $\zeta_{0} \notin S_{g}$; hence

$$
0 \geq \Delta \log f_{r}\left(\zeta_{0}\right) \geq \Delta \log g\left(\zeta_{0}\right)-\Delta \log g_{a}^{r}\left(\zeta_{0}\right)=-2 g\left(\zeta_{0}\right) K\left(\mu_{g}\right)\left(\zeta_{0}\right)-8 a g_{a}^{r}\left(\zeta_{0}\right)
$$

By assumption, $K\left(\mu_{g}\right)\left(\zeta_{0}\right) \leq-4 a$; therefore (1.5) yields $g\left(\zeta_{0}\right) \leq g_{a}^{r}\left(\zeta_{0}\right)$ and thus, by the choice of $\zeta_{0}$,

$$
\forall \zeta \in U_{r} \quad g(\zeta) \leq g_{a}(\zeta / r)
$$

Letting $r \rightarrow 1^{-}$we obtain the assertion.

To complement this result, we recall a theorem due to Heins [H, Theorem 7.1], showing that $\mu_{g} \neq \mu_{a}$ in the statement of Proposition 1.1 implies that $g$ is strictly less than $g_{a}$ everywhere:

Theorem 1.2: (Heins) Let $\mu_{g}=g d \zeta \otimes d \bar{\zeta}$ be a pseudohermitian metric on $U$ such that $K\left(\mu_{g}\right) \leq-4 a$ on $U \backslash S_{g}$ for some $a>0$. Assume there is $\zeta_{0} \in U \backslash S_{g}$ such that $g\left(\zeta_{0}\right)=g_{a}\left(\zeta_{0}\right)$. Then $\mu_{g} \equiv \mu_{a}$.

Now we start looking to the several variables situation. If $M$ is a complex manifold, we shall denote by $T M$ its real tangent bundle endowed with the almost-complex structure $J$ induced by the complex structure of $M$; by $T^{c} M$ the complexification of $T M$ and by $T^{1,0} M$ the (1,0)-part (i.e., the $i$-eigenspace of $J$ ) of $T^{c} M$. As well known, $T^{1,0} M$ is naturally complex-isomorphic to $T M$. In this paper we shall mainly use $T^{1,0} M$ as representative of the tangent bundle of $M$, except for an argument needed in section 3 . 
A complex Finsler metric $F$ on a complex $n$-dimensional $(n \geq 1)$ manifold $M$ is an upper semicontinuous map $F: T^{1,0} M \rightarrow \mathbb{R}^{+}$satisfying

(i) $F(p ; v)>0$ for all $p \in M$ and $v \in T_{p}^{1,0} M$ with $v \neq 0$;

(ii) $F(p ; \lambda v)=|\lambda| F(p ; v)$ for all $p \in M, v \in T_{p}^{1,0} M$ and $\lambda \in \mathbb{C}$.

We shall sistematically denote by $G: T^{1,0}(M) \rightarrow \mathbb{R}^{+}$the function $G=F^{2}$. Note that, thanks to condition (ii), the definition of length of a smooth curve in a Riemannian manifold makes sense in this context too; so we may again associate to $F$ a topological distance on $M$, and we shall say that $F$ is complete if this distance is. For the same reason, it makes sense to call (real) geodesics the extremals of the length functional. General introductions to real Finsler geometry are [Ru1, B].

Take $p \in M$ and $v \in T_{p}^{1,0} M, v \neq 0$. The holomorphic curvature $K_{F}(p ; v)$ of $F$ at $(p ; v)$ is given by

$$
K_{F}(p ; v)=\sup \left\{K\left(\varphi^{*} G\right)(0)\right\},
$$

where the supremum is taken with respect to the family of all holomorphic maps $\varphi: U \rightarrow M$ with $\varphi(0)=p$ and $\varphi^{\prime}(0)=\lambda v$ for some $\lambda \in \mathbb{C}^{*}$, and $K\left(\varphi^{*} G\right)$ is the Gaussian curvature discussed so far of the pseudohermitian metric $\varphi^{*} G$ on $U$. Clearly, the holomorphic curvature depends only on the complex line in $T_{p}^{1,0} M$ spanned by $v$, and not on $v$ itself.

The holomorphic curvature may also be defined (see e.g. [S]) taking the supremum with respect to the family of all holomorphic maps $\varphi: U_{r} \rightarrow M$ with $\varphi(0)=p$ and $\varphi^{\prime}(0)=v$, where $U_{r} \subset \mathbb{C}$ is the disk of center the origin and radius $r$. We chose the given definition to stress the similarities with the definitions of the Kobayashi and Carathéodory metrics.

If $F$ is a complex Finsler metric on $U$ - and so $G=F^{2}$ is a pseudohermitian metric $G=g d \zeta \otimes d \bar{\zeta}$ on $U-$, a priori we have defined two curvatures for $F: K_{F}(\zeta ; 1)$ and $K(G)(\zeta)$. As anybody may guess, they actually coincide; this is a consequence of

Lemma 1.3: Let $\mu_{g}=g d \zeta \otimes d \bar{\zeta}$ be a pseudohermitian metric on $U$, and $\varphi: U \rightarrow U$ a holomorphic self-map of $U$. Then on $U \backslash\left[\varphi^{-1}\left(S_{g}\right) \cup S_{\varphi^{\prime}}\right]$

$$
K\left(\varphi^{*} \mu_{g}\right)=K\left(\mu_{g}\right) \circ \varphi
$$

Proof: Let $\left\{g_{n}\right\}$ be a sequence of $C^{2}$ functions such that $g_{n} \geq g_{n+1}$ and with $g_{n}(x) \rightarrow g(x)$. Then on $U \backslash\left[\varphi^{-1}\left(S_{g_{n}}\right) \cup S_{\varphi^{\prime}}\right] \supset U \backslash\left[\varphi^{-1}\left(S_{g}\right) \cup S_{\varphi^{\prime}}\right]$ we have

$$
\begin{aligned}
K\left(\varphi^{*} \mu_{g_{n}}\right) & =-\frac{1}{2\left|\varphi^{\prime}\right|^{2}\left(g_{n} \circ \varphi\right)} \Delta \log \left(\left|\varphi^{\prime}\right|^{2} g_{n} \circ \varphi\right) \\
& =-\frac{2}{\left|\varphi^{\prime}\right|^{2}\left(g_{n} \circ \varphi\right)}\left[\frac{\partial^{2} \log \left(g_{n} \circ \varphi\right)}{\partial \zeta \partial \bar{\zeta}}+\frac{\partial^{2} \log \left|\varphi^{\prime}\right|^{2}}{\partial \zeta \partial \bar{\zeta}}\right] \\
& =-\frac{2}{\left|\varphi^{\prime}\right|^{2}\left(g_{n} \circ \varphi\right)}\left[\left|\varphi^{\prime}\right|^{2}\left(\frac{\partial^{2} \log g_{n}}{\partial \zeta \partial \bar{\zeta}}\right) \circ \varphi\right] \\
& =-\frac{1}{2\left(g_{n} \circ \varphi\right)}\left(\Delta \log g_{n}\right) \circ \varphi=K\left(\mu_{g_{n}}\right) \circ \varphi .
\end{aligned}
$$

Letting $n \rightarrow+\infty$ and applying the dominated convergence theorem we get the assertion. 
The holomorphic curvature defined in this way is clearly invariant under holomorphic isometries. More generally, if $f: M \rightarrow N$ is holomorphic and $F$ is a Finsler metric on $N$, we have

$$
K_{F}\left(f(z) ; d f_{z}(v)\right) \geq K_{f^{*} F}(z ; v),
$$

that is $f^{*} K_{F} \geq K_{f^{*} F}$.

When $F$ is a honest smooth hermitian metric on $M, K_{F}(p ; v)$ coincides with the usual holomorphic sectional curvature of $F$ at $(p ; v)$ (see $[\mathrm{Wu}]$ ). The aim of this section is to extend a couple of results already known for hermitian metrics to this more general case.

A piece of terminology: we say that a complex Finsler metric $F$ has holomorphic curvature bounded above (below) by a constant $c \in \mathbb{R}$ if $K_{F}(p ; v) \leq c$ (respectively, $\left.K_{F}(p ; v) \geq c\right)$ for all $(p ; v) \in T^{1,0} M$ with $v \neq 0$.

Our first result is the usual several variables version of Ahlfors' lemma:

Proposition 1.4: Let $F$ be a complex Finsler metric on a complex manifold $M$. Assume that the holomorphic curvature of $F$ is bounded above by a negative constant $-4 a$, for some $a>0$. Then

$$
\varphi^{*} F \leq \mu_{a}
$$

for all holomorphic maps $\varphi: U \rightarrow M$.

Proof: $\varphi^{*} F$ is a pseudohermitian metric on $U$; by assumption (and by Lemma 1.3), $K\left(\varphi^{*} F\right) \leq-4 a$. Then the assertion follows from Proposition 1.1.

As a consequence, we obtain a generalization of a well-known criterion of hyperbolicity:

Corollary 1.5: Let $M$ be a complex manifold admitting a (complete) complex Finsler metric $F$ with holomorphic curvature bounded above by a negative constant. Then $M$ is (complete) hyperbolic.

Proof: Up to multiplying $F$ by a suitable constant, we may assume $K_{F} \leq-4$. Let $d$ denote the distance induced by $F$ on $M$, and $\omega$ the Poincaré distance on $U$. Then Proposition 1.4 yields

$$
d\left(\varphi\left(\zeta_{1}\right), \varphi\left(\zeta_{2}\right)\right) \leq \omega\left(\zeta_{1}, \zeta_{2}\right)
$$

for all $\zeta_{1}, \zeta_{2} \in U$ and holomorphic maps $\varphi: U \rightarrow M$. But this immediately implies (cf. [K1, Proposition IV.1.4]) that the Kobayashi distance $k_{M}$ of $M$ is bounded below by $d$, and the assertion follows.

In particular, then, a complex manifold admitting a complete complex Finsler metric with holomorphic curvature bounded above by a negative constant is necessarily taut.

The notion of holomorphic curvature for (non-smooth) Finsler metric has been introduced recently in connection with the Carathéodory and Kobayashi metrics. In particular, Wong [W] and Suzuki [S] (see also [Bu]) have shown that the holomorphic curvature of the Carathéodory metric is bounded above by -4 , whereas the holomorphic curvature of the Kobayashi metric is bounded below by -4 .

An interesting immediate consequence of this is an interpretation in terms of curvature of a well known property of the Carathéodory metric: 
Proposition 1.6: Let $F$ be a complex Finsler metric on a manifold $M$ with holomorphic curvature bounded above by -4 . Let $\varphi: U \rightarrow M$ be a holomorphic map. Then the following are equivalent:

(i) $\varphi^{*} F(0 ; 1)=F\left(\varphi(0) ; \varphi^{\prime}(0)\right)=1$, that is $\varphi$ is an isometry at the origin between the Poincaré metric on $U$ and $F$;

(ii) $\varphi$ is an infinitesimal complex geodesic, that is $\varphi^{*} F$ is the Poincaré metric of $U$.

Proof: By definition and Lemma 1.3, the Gaussian curvature of $\varphi^{*} F$ is bounded above by -4 . The assertion follows from Heins' Theorem 1.2.

Bounds on the holomorphic curvature allow to compare a complex Finsler metric to the Kobayashi metric - and maybe to prove that a given Finsler metric actually is the Kobayashi metric. For instance, Pang $[\mathrm{P}]$ and Faran $[\mathrm{F}]$ gave conditions under which a smooth complex Finsler metric of constant negative holomorphic curvature coincides with the Kobayashi metric. We shall discuss the smooth case in detail in the next two sections; here, to provide the right set-up to the problem, we examine a bit the general situation.

We need an auxiliary notion to formulate our observation. Let $F$ be a complex Finsler metric on a manifold $M$, and take $(p ; v) \in T^{1,0} M$. We say that $F$ is realizable at $(p ; v)$ if there is a holomorphic map $\varphi: U \rightarrow M$ such that $\varphi(0)=p$ and $\lambda \varphi^{\prime}(0)=v$ with $|\lambda|=F(p ; v)$. In other words, $\varphi$ is an isometry at the origin between the Poincaré metric of $U$ and $F$.

Obviously, the Kobayashi metric is realizable in any taut manifold; on the other hand, as a consequence of the next result, the Carathéodory metric is realizable iff it coincides with the Kobayashi metric.

Proposition 1.7: Let $F$ be a complex Finsler metric on a manifold $M$, and choose $\left(p_{0} ; v_{0}\right) \in T^{1,0} M$. Then:

(i) If $F$ is realizable at $\left(p_{0} ; v_{0}\right)$, then $F\left(p_{0} ; v_{0}\right) \geq \kappa_{M}\left(p_{0} ; v_{0}\right)$;

(ii) If $K_{F} \leq-4$, then $F \leq \kappa_{M}$;

(iii) If $F$ is realizable at $\left(p_{0} ; v_{0}\right)$ and $K_{F} \leq-4$, then $F\left(p_{0} ; v_{0}\right)=\kappa_{M}\left(p_{0} ; v_{0}\right)$.

Proof: (i) Let $\varphi: U \rightarrow M$ be a holomorphic map with $\varphi(0)=p_{0}$ and $v_{0}=\lambda \varphi^{\prime}(0)$ such that $F\left(p_{0} ; v_{0}\right)=|\lambda|$. Then

$$
F\left(p_{0} ; v_{0}\right)=|\lambda| \geq \kappa_{M}\left(p_{0} ; v_{0}\right) .
$$

(ii) Take $(p ; v) \in T^{1,0} M$ and let $\varphi: U \rightarrow M$ be a holomorphic map with $\varphi(0)=p$ and $v=\lambda \varphi^{\prime}(0)$. Then $\varphi^{*} G$ is a pseudohermitian metric on $U$ with Gaussian curvature bounded above by -4 (by Lemma 1.3); it follows from Proposition 1.1 that $\varphi^{*} G \leq \mu_{1}$. Thus

$$
F(p ; v)=F\left(\varphi(0) ; \lambda \varphi^{\prime}(0)\right)=\varphi^{*} F(0 ; \lambda) \leq|\lambda| .
$$

Since this holds for all such $\varphi$, we get $F \leq \kappa_{M}$.

(iii) Obvious, now.

This is the best it can be done on the basis of Ahlfors' lemma and Heins' theorem only. In order to get deeper results it is necessary to use more tools - as we shall see in the smooth case discussed in the rest of the paper. 


\section{Holomorphic curvature: the smooth case}

In this section we shall derive a tensor expression of the holomorphic curvature of a smooth complex Finsler metric.

First of all, we need a few definitions, notations and general formulas. Let $F$ be a complex Finsler metric on a complex manifold $M$, and set $G=F^{2}$, as usual. We shall assume that $F$ is smooth, that is that $F$ is of class $C^{k}(k \geq 4)$ out of the zero section of $T^{1,0} M$. By the way, $\left(T^{1,0} M\right)_{0}$ will denote the complement in $T^{1,0} M$ of the zero section.

If $\left(z^{1}, \ldots, z^{n}\right)$ are local coordinates on $M$, a local section of $T^{1,0} M$ will be written as

$$
\sum_{j=1}^{n} v^{j} \frac{\partial}{\partial z^{j}}
$$

and we shall use $\left(z^{1}, \ldots, z^{n} ; v^{1}, \ldots, v^{n}\right)$ as local coordinates on $T^{1,0} M$.

We shall denote by indexes like $\alpha, \bar{\beta}$ and so on the derivatives with respect to the $v$-coordinates; for instance,

$$
G_{\alpha \bar{\beta}}=\frac{\partial^{2} G}{\partial v^{\alpha} \partial \overline{v^{\beta}}} .
$$

On the other hand, the derivatives with respect to the $z$-coordinates will be denoted by indexes after a semicolon; for instance,

$$
G_{; i j}=\frac{\partial^{2} G}{\partial z^{i} \partial z^{j}} \quad \text { or } \quad G_{\alpha ; \bar{j}}=\frac{\partial^{2} G}{\partial \overline{z^{j}} \partial v^{\alpha}} .
$$

A smooth complex Finsler metric $F$ will be said strongly pseudoconvex if the $F$ indicatrices are strongly pseudoconvexes, i.e., if the Levi matrix $\left(G_{\alpha \bar{\beta}}\right)$ is positive definite on $\left(T^{1,0} M\right)_{0}$. As usual in hermitian geometry, we shall denote by $\left(G^{\alpha \bar{\beta}}\right)$ the inverse matrix of $\left(G_{\alpha \bar{\beta}}\right)$, and we shall use it to raise indexes. The usual summation convention will hold throughout the rest of the paper.

The main (actually, almost the unique) property of the function $G$ is its (1,1)-homogeneity: we have

$$
G(z ; \lambda v)=\lambda \bar{\lambda} G(z ; v)
$$

for all $(z ; v) \in T^{1,0} M$ and $\lambda \in \mathbb{C}$. We now collect a number of formulas we shall use later on which follows from (2.1). First of all, differentiating with respect to $v^{\alpha}$ and $\overline{v^{\beta}}$ we get

$$
\begin{aligned}
G_{\alpha}(z ; \lambda v) & =\bar{\lambda} G_{\alpha}(z ; v), \\
G_{\alpha \bar{\beta}}(z ; \lambda v) & =G_{\alpha \bar{\beta}}(z ; v), \\
G_{\alpha \beta}(z ; \lambda v) & =(\bar{\lambda} / \lambda) G_{\alpha \beta}(z ; v) .
\end{aligned}
$$

Thus differentiating with respect to $\lambda$ or $\bar{\lambda}$ and then setting $\lambda=1$ we get

$$
G_{\alpha \bar{\beta}} \overline{v^{\beta}}=G_{\alpha}, \quad G_{\alpha \beta} v^{\beta}=0
$$


and

$$
G_{\alpha \beta \gamma} v^{\gamma}=-G_{\alpha \beta}, \quad G_{\alpha \beta \bar{\gamma}} \overline{v^{\gamma}}=G_{\alpha \beta}, \quad G_{\alpha \bar{\beta} \gamma} v^{\gamma}=0,
$$

where everything is evaluated at $(z ; v)$.

On the other hand, differentiating directly (2.1) with respect to $\lambda$ or $\bar{\lambda}$ and putting eventually $\lambda=1$ we get

$$
G_{\alpha} v^{\alpha}=G, \quad G_{\alpha \beta} v^{\alpha} v^{\beta}=0, \quad G_{\alpha \bar{\beta}} v^{\alpha} \overline{v^{\beta}}=G .
$$

It is clear that we may get other formulas applying any differential operator acting only on the $z$-coordinates, or just by conjugation. For instance, we get

$$
G_{\bar{\alpha} ; i} \overline{v^{\alpha}}=G_{; i}
$$

and so on.

Assuming now $F$ strongly pseudoconvex, we get another bunch of formulas we shall need later on. First of all, applying $G^{\alpha \bar{\beta}}$ to the first equation in (2.2) we get

$$
G^{\alpha \bar{\beta}} G_{\alpha}=\overline{v^{\beta}}
$$

and thus, applying (2.5),

$$
G_{\bar{\beta} ; i} G^{\alpha \bar{\beta}} G_{\alpha}=G_{; i} .
$$

Recalling that $\left(G^{\alpha \bar{\beta}}\right)$ is the inverse matrix of $\left(G_{\alpha \bar{\beta}}\right)$, we may also compute derivatives of $G^{\alpha \bar{\beta}}$ :

$$
D G^{\alpha \bar{\beta}}=-G^{\alpha \bar{\nu}} G^{\mu \bar{\beta}}\left(D G_{\mu \bar{\nu}}\right),
$$

where $D$ denotes any first order linear differential operator. As a consequence of $(2.3)$ and $(2.8)$ we get

$$
G_{\bar{\sigma}}^{\alpha \bar{\beta}} \overline{v^{\sigma}}=-G^{\alpha \bar{\nu}} G^{\mu \bar{\beta}} G_{\mu \bar{\nu} \bar{\sigma}} \overline{v^{\sigma}}=0,
$$

and recalling also (2.6) we obtain

$$
G_{\bar{\beta}} G_{\gamma}^{\alpha \bar{\beta}}=-G_{\bar{\beta}} G^{\mu \bar{\beta}} G^{\alpha \bar{\nu}} G_{\mu \bar{\nu} \gamma}=-G^{\alpha \bar{\nu}} G_{\mu \bar{\nu} \gamma} v^{\mu}=0 .
$$

Now we may start to work. Our first goal is to compute the holomorphic curvature of our strongly pseudoconvex smooth complex Finsler metric $F$. Set

$$
S^{1,0} M=\left\{\xi \in T^{1,0} M \mid F(\xi)=1\right\},
$$

and choose $p \in M$ and $\xi \in S_{p}^{1,0} M$. To compute $K_{F}(p ; \xi)$ we should write the Gaussian curvature at the origin of $\varphi^{*} G$, where $\varphi: U \rightarrow M$ is any holomorphic map with $\varphi(0)=p$ and $v=\varphi^{\prime}(0)=\lambda \xi$, where $|\lambda|=F(p ; v)=\left[\varphi^{*} G(0 ; 1)\right]^{1 / 2}$.

Writing $\varphi^{*} G=g d \zeta \otimes d \bar{\zeta}$, we have

$$
g(\zeta)=G\left(\varphi(\zeta) ; \varphi^{\prime}(\zeta)\right), \quad g(0)=|\lambda|^{2},
$$


and

$$
K\left(\varphi^{*} G\right)(0)=-\frac{1}{2 g(0)}(\Delta \log g)(0)=-\frac{2}{|\lambda|^{2}} \frac{\partial^{2}(\log g)}{\partial \bar{\zeta} \partial \zeta}(0) .
$$

The computation of the Laplacian yields

$$
\begin{aligned}
\frac{\partial^{2}(\log g)}{\partial \bar{\zeta} \partial \zeta}= & -\frac{1}{G\left(\varphi ; \varphi^{\prime}\right)^{2}}\left|G_{; i}\left(\varphi ; \varphi^{\prime}\right)\left(\varphi^{\prime}\right)^{i}+G_{\alpha}\left(\varphi ; \varphi^{\prime}\right)\left(\varphi^{\prime \prime}\right)^{\alpha}\right|^{2} \\
& +\frac{1}{G\left(\varphi ; \varphi^{\prime}\right)}\left\{G_{; i \bar{j}}\left(\varphi ; \varphi^{\prime}\right)\left(\varphi^{\prime}\right)^{i} \overline{\left(\varphi^{\prime}\right)^{j}}+G_{\alpha \bar{\beta}}\left(\varphi ; \varphi^{\prime}\right)\left(\varphi^{\prime \prime}\right)^{\alpha} \overline{\left(\varphi^{\prime \prime}\right)^{\beta}}\right. \\
& \left.+2 \operatorname{Re}\left[G_{\bar{\alpha} ; i}\left(\varphi ; \varphi^{\prime}\right)\left(\varphi^{\prime}\right)^{i} \overline{\left(\varphi^{\prime \prime}\right)^{\alpha}}\right]\right\} .
\end{aligned}
$$

Hence writing $\eta=\varphi^{\prime \prime}(0)$ we get

$$
\begin{aligned}
K\left(\varphi^{*} G\right)(0)= & -2\left[G_{; i \bar{\jmath}}(p ; \xi)-G_{; i}(p ; \xi) G_{; \bar{j}}(p ; \xi)\right] \xi^{i} \overline{\xi^{j}} \\
& -\frac{2}{|\lambda|^{4}}\left[G_{\alpha \bar{\beta}}(p ; \xi)-G_{\alpha}(p ; \xi) G_{\bar{\beta}}(p ; \xi)\right] \eta^{\alpha} \overline{\eta^{\beta}} \\
& -\frac{4}{|\lambda|^{4}} \operatorname{Re}\left\{\lambda^{2}\left[G_{\bar{\alpha} ; i}(p ; \xi)-G_{\bar{\alpha}}(p ; \xi) G_{; i}(p ; \xi)\right] \xi^{i} \overline{\eta^{\alpha}}\right\} .
\end{aligned}
$$

We must compute the supremum (with respect to $\lambda$ and $\eta$ ) of this formula. For the moment, let us consider $\lambda$ fixed, and look for the infimum of

$$
\left[G_{\alpha \bar{\beta}}(p ; \xi)-G_{\alpha}(p ; \xi) G_{\bar{\beta}}(p ; \xi)\right] \eta^{\alpha} \overline{\eta^{\beta}}+2 \operatorname{Re}\left\{\lambda^{2}\left[G_{\bar{\alpha} ; i}(p ; \xi)-G_{\bar{\alpha}}(p ; \xi) G_{; i}(p ; \xi)\right] \xi^{i} \overline{\eta^{\alpha}}\right\}
$$

that is of

$$
I_{\lambda}(\eta)=A_{\alpha \bar{\beta}} \eta^{\alpha} \overline{\eta^{\beta}}+2 \operatorname{Re}\left[\lambda^{2} B_{\bar{\alpha} ; i} \xi^{i} \overline{\eta^{\alpha}}\right]
$$

where

$$
\begin{aligned}
& A_{\alpha \bar{\beta}}=G_{\alpha \bar{\beta}}(p ; \xi)-G_{\alpha}(p ; \xi) G_{\bar{\beta}}(p ; \xi), \\
& B_{\bar{\alpha} ; i}=G_{\bar{\alpha} ; i}(p ; \xi)-G_{\bar{\alpha}}(p ; \xi) G_{; i}(p ; \xi) .
\end{aligned}
$$

Let us study the hermitian form $\left(A_{\alpha \bar{\beta}}\right)$. By assumption, the matrix $\left(G_{\alpha \bar{\beta}}(p ; \xi)\right)$ induces a positive definite hermitian product on $\mathbb{C}^{n}$; so we may decompose $\mathbb{C}^{n}$ accordingly as the orthogonal sum of $\mathbb{C} \xi$ and its orthogonal $(\mathbb{C} \xi)^{\perp}$. Since, by $(2.4)$ and $(2.5)$,

$$
\begin{gathered}
A_{\alpha \bar{\beta}} \eta^{\alpha} \overline{\xi^{\beta}}=G_{\alpha \bar{\beta}} \eta^{\alpha \overline{\xi^{\beta}}}-G_{\alpha} G_{\bar{\beta}} \eta^{\alpha} \overline{\xi^{\beta}}=G_{\alpha} \eta^{\alpha}-G_{\alpha} \eta^{\alpha}=0 \\
B_{\bar{\alpha} ; i} \xi^{i \overline{\xi^{\alpha}}}=G_{\bar{\alpha} ; i} \xi^{i \overline{\xi^{\alpha}}}-G_{\bar{\alpha}} G_{; i} \xi^{i} \overline{\xi^{\alpha}}=G_{; i} \xi^{i}-G_{; i} \xi^{i}=0
\end{gathered}
$$

for every $\eta \in \mathbb{C}^{n}$, if we denote by

$$
\tilde{\eta}=\eta-\left(G_{\alpha \bar{\beta}} \eta^{\alpha} \overline{\xi^{\beta}}\right) \xi
$$

the orthogonal projection of $\eta$ into $(\mathbb{C} \xi)^{\perp}$, we get 
Lemma 2.1: Let $F$ be a strongly pseudoconvex smooth complex Finsler metric on $M$, and take $p \in M$ and $\xi \in S_{p}^{1,0} M$. Then $I_{\lambda} \equiv 0$ on $\mathbb{C} \xi$ and $I_{\lambda}(\tilde{\eta})=I_{\lambda}(\eta)$ for all $\eta \in \mathbb{C}^{n}$.

So it suffices to study $I_{\lambda}$ on $(\mathbb{C} \xi)^{\perp}$. Note that $\tilde{\eta} \in(\mathbb{C} \xi)^{\perp}$ iff

$$
0=G_{\alpha \bar{\beta}} \tilde{\eta}^{\alpha} \overline{\xi^{\beta}}=G_{\alpha} \tilde{\eta}^{\alpha}
$$

therefore on $(\mathbb{C} \xi)^{\perp}$ we have

$$
A_{\alpha \bar{\beta}} \tilde{\eta}^{\alpha}=G_{\alpha \bar{\beta}} \tilde{\eta}^{\alpha}
$$

In particular $\left(A_{\alpha \bar{\beta}}\right)$ is positive definite on $(\mathbb{C} \xi)^{\perp}$.

Thus $I_{\lambda}$ is a quadratic polynomial on $(\mathbb{C} \xi)^{\perp}$ with positive definite leading term; hence $I_{\lambda}$ attains a minimum at $\tilde{\eta} \in(\mathbb{C} \xi)^{\perp}$ given by

$$
A_{\alpha \bar{\beta}} \tilde{\eta}^{\alpha}=-\lambda^{2} B_{\bar{\beta} ; i} \xi^{i}, \quad \beta=1, \ldots, n,
$$

that is, by $(2.13)$,

$$
\tilde{\eta}^{\alpha}=-\lambda^{2} G^{\alpha \bar{\beta}} B_{\bar{\beta} ; i} \xi^{i}, \quad \alpha=1, \ldots, n .
$$

Putting (2.14) into (2.12) we find that the minimum of $I_{\lambda}$ is

$$
-|\lambda|^{4} G^{\alpha \bar{\beta}}\left(B_{\alpha ; \bar{j}} \overline{\xi^{j}}\right)\left(B_{\bar{\beta} ; i} \xi^{i}\right)<0,
$$

and thus (2.11) yields

$$
K_{F}(p ; \xi)=-2\left[G_{; i \bar{\jmath}}-G_{; i} G_{; \bar{\jmath}}-G^{\alpha \bar{\beta}} B_{\alpha ; \bar{\jmath}} B_{\bar{\beta} ; i}\right] \xi^{i} \overline{\xi^{j}}
$$

Now, using (2.4) and (2.6) we get

$$
G^{\alpha \bar{\beta}} B_{\alpha ; \bar{j}} B_{\bar{\beta} ; i}=G^{\alpha \bar{\beta}} G_{\alpha ; \bar{\jmath}} G_{\bar{\beta} ; i}-G_{; i} G_{; \bar{j}} ;
$$

therefore

$$
K_{F}(p ; \xi)=-2\left[G_{; i \bar{\jmath}}-G^{\alpha \bar{\beta}} G_{\alpha ; \bar{\jmath}} G_{\bar{\beta} ; i}\right] \xi^{i} \overline{\xi^{j}} .
$$

It is easy to check (cf. [Wu]) that when $F$ is a standard hermitian metric on $M$, then (2.15) reduces to the usual holomorphic sectional curvature in the direction $\xi$. Furthermore, (2.15) exactly yields the holomorphic sectional curvature introduced by Kobayashi in [K2].

There is a shorter way of writing $K_{F}$. Set

$$
\Gamma_{; i}^{\alpha}=G^{\alpha \bar{\mu}} G_{\bar{\mu} ; i}
$$

and put $\Gamma_{; i \bar{\jmath}}^{\alpha}=\left(\Gamma_{; i}^{\alpha}\right)_{; \bar{\jmath}}$; then, by $(2.8)$,

$$
\Gamma_{; i \bar{\jmath}}^{\alpha}=G^{\alpha \bar{\mu}} G_{\bar{\mu} ; i \bar{\jmath}}-G^{\alpha \bar{\nu}} G^{\beta \bar{\mu}} G_{\beta \bar{\nu} ; \bar{\jmath}} G_{\bar{\mu} ; i},
$$

and so, by (2.6) and (2.7),

$$
G_{\alpha} \Gamma_{; i \bar{\jmath}}^{\alpha}=G_{; i \bar{\jmath}}-G^{\beta \bar{\mu}} G_{\beta ; \bar{\jmath}} G_{\bar{\mu} ; i} .
$$

Summing up, we have proved the 
Proposition 2.2: Let $F$ be a strongly pseudoconvex smooth complex Finsler metric on $M$, and take $(p ; \xi) \in S^{1,0} M$. Then the holomorphic curvature of $F$ in the direction of $\xi$ is

$$
K_{F}(p ; \xi)=-2 G_{\alpha} \Gamma_{; i \bar{\jmath}}^{\alpha} \xi^{i} \overline{\xi^{j}} .
$$

For future reference, we note here that more generally the holomorphic curvature of $F$ in the direction of a non-zero vector $v \in T_{p}^{1,0} M$ - which coincides with the holomorphic curvature in the direction of $\xi=v / F(p ; v)$ - is given by the formula

$$
K_{F}(p ; v)=-\frac{2}{G(p ; v)^{2}} G_{\alpha}(p ; v) \Gamma_{; i \bar{\jmath}}^{\alpha}(p ; v) v^{i} \overline{v^{j}} .
$$

\section{Holomorphic curvature and geodesic complex curves}

Let $F$ be a (smooth) complex Finsler metric on a manifold $M$. Let $U_{r}$ denote the disk $\{\zeta \in \mathbb{C}|| \zeta \mid<r\}$ in $\mathbb{C}$ (with $0<r \leq 1$ ), endowed with the restriction of the Poincaré metric of $U$; note that $U_{r}$ is a convex subset of $U$ with respect to the Poincaré metric. A holomorphic map $\varphi: U_{r} \rightarrow M$ is a segment of infinitesimal complex geodesic if $\varphi^{*} F$ is the Poincaré metric on $U_{r}$, that is if $\varphi$ is a local isometry from the Poincaré metric to $F$. On the other hand, $\varphi$ is said segment of geodesic complex curve if the image via $\varphi$ of any (real) geodesic in $U_{r}$ is a (real) geodesic for $F$ in $M$. In other words, $\varphi$ is a local isometry and $\varphi\left(U_{r}\right)$ is a totally geodesic complex curve in $M$. When $r=1$, we shall talk of infinitesimal complex geodesics and geodesic complex curves tout-court. In any case, if $(p ; v)=\left(\varphi(0) ; \varphi^{\prime}(0)\right)$ we say that $\varphi$ is tangent to $(p ; v)$.

In $[\mathrm{AP}]$ we showed that $\varphi$ is a segment of geodesic complex curve iff it is a holomorphic solution of the system

$$
\begin{gathered}
\left(\varphi^{\prime \prime}\right)^{\alpha}+A\left(\varphi^{\prime}\right)^{\alpha}=-\Gamma_{; i}^{\alpha}\left(\varphi ; \varphi^{\prime}\right)\left(\varphi^{\prime}\right)^{i}, \quad \alpha=1, \ldots, n, \\
G_{\alpha \beta}\left(\varphi ; \varphi^{\prime}\right)\left(\varphi^{\prime \prime}+A \varphi^{\prime}\right)^{\beta}=\left[G_{i ; \alpha}\left(\varphi ; \varphi^{\prime}\right)-G_{\alpha ; i}\left(\varphi ; \varphi^{\prime}\right)\right]\left(\varphi^{\prime}\right)^{i}, \quad \alpha=1, \ldots, n,
\end{gathered}
$$

where the prime stands for $\partial / \partial \zeta$, and $A: U \rightarrow \mathbb{C}$ is the function

$$
A(\zeta)=-\frac{2 \bar{\zeta}}{1-|\zeta|^{2}}
$$

As we shall see later on, the main amount of informations is contained in equation (3.1). For the moment, however, let us discuss equation (3.2) a bit.

Let $\varphi: U_{r} \rightarrow M$ be a holomorphic solution of equation (3.1). Then, putting (3.1) into (3.2), we find that $\varphi$ is a segment of geodesic complex curve iff

$$
G_{\alpha \beta}\left(\varphi ; \varphi^{\prime}\right) \Gamma_{; i}^{\beta}\left(\varphi ; \varphi^{\prime}\right)\left(\varphi^{\prime}\right)^{i}=\left[G_{\alpha ; i}\left(\varphi ; \varphi^{\prime}\right)-G_{; \alpha}\left(\varphi ; \varphi^{\prime}\right)\right]\left(\varphi^{\prime}\right)^{i}
$$

that is iff along the curve $\varphi$ we have

$$
\left[G_{i ; \alpha}-G_{\alpha ; i}+G_{\alpha \beta} \Gamma_{; i}^{\beta}\right] v^{i}=0, \quad \alpha=1, \ldots, n .
$$


In a more symmetric way, following [Ru2] we may introduce the torsion tensor

$$
T_{\alpha i \bar{\mu}}=\left(G_{i \bar{\mu} ; \alpha}-G_{i \bar{\mu} \beta} \Gamma_{; \alpha}^{\beta}\right)-\left(G_{\alpha \bar{\mu} ; i}-G_{\alpha \bar{\mu} \beta} \Gamma_{; i}^{\beta}\right) ;
$$

it is a $(3,0)$-tensor defined on $\left(T^{1,0} M\right)_{0}$. Then $(3.3)$ is equivalent to

$$
T_{\alpha i \bar{\mu}} \overline{v^{\mu}} v^{i}=0, \quad \alpha=1, \ldots, n .
$$

If $G(p ; v)=g_{\alpha \bar{\beta}}(p) v^{\alpha} v^{\beta}$ is a standard hermitian metric, then $G_{\alpha \beta} \equiv 0$ and (3.3) reduces to

$$
\frac{\partial g_{\alpha \bar{\mu}}}{\partial z^{i}}=\frac{\partial g_{i \bar{\mu}}}{\partial z^{\alpha}}
$$

that is to the usual Kähler condition. For this reason, a strongly pseudoconvex smooth complex Finsler metric satisfying (3.3) will be said Kähler. Summing up, we have proved

Proposition 3.1: Let $F$ be a strongly pseudoconvex smooth complex Finsler metric on a manifold $M$. Then a holomorphic solution $\varphi$ of (3.1) is a segment of geodesic complex curve iff $F$ is Kähler along $\varphi$.

It is possible to write (3.3) in still another way. Set $\Gamma_{\beta ; i}^{\alpha}=\left(\Gamma_{; i}^{\alpha}\right)_{\beta}$; then, using $(2.3)$, (2.6) and (2.8),

$$
\Gamma_{\beta ; i}^{\alpha}=-G^{\gamma \bar{\mu}} G^{\alpha \bar{\nu}} G_{\gamma \bar{\nu} \beta} G_{\bar{\mu} ; i}+G^{\alpha \bar{\mu}} G_{\beta \bar{\mu} ; i}
$$

and so

$$
\begin{gathered}
G_{\alpha} \Gamma_{\beta ; i}^{\alpha}=G_{\beta ; i}-G_{\beta \gamma} \Gamma_{; i}^{\gamma}, \\
G_{\alpha \bar{\mu}} \Gamma_{\beta ; i}^{\alpha}=G_{\beta \bar{\mu} ; i}-G_{\beta \bar{\mu} \gamma} \Gamma_{; i}^{\gamma}, \\
G_{\alpha} \Gamma_{i ; \beta}^{\alpha} v^{i}=G_{i ; \beta} v^{i} .
\end{gathered}
$$

Then

$$
\begin{gathered}
T_{\alpha i \bar{\mu}}=G_{\beta \bar{\mu}}\left(\Gamma_{i ; \alpha}^{\beta}-\Gamma_{\alpha ; i}^{\beta}\right), \\
{\left[G_{i ; \alpha}-G_{\alpha ; i}+G_{\alpha \beta} \Gamma_{; i}^{\beta}\right] v^{i}=G_{\beta}\left(\Gamma_{i ; \alpha}^{\beta}-\Gamma_{\alpha ; i}^{\beta}\right) v^{i},}
\end{gathered}
$$

and (3.3) is equivalent to $G_{\beta}\left(\Gamma_{i ; \alpha}^{\beta}-\Gamma_{\alpha ; i}^{\beta}\right) v^{i}=0$ for $\alpha=1, \ldots, n$. We remark that when $G$ is a hermitian metric then

$$
\Gamma_{\beta ; i}^{\alpha}=g^{\alpha \bar{\mu}} \frac{\partial g_{\beta \bar{\mu}}}{\partial z^{i}},
$$

and so they are the coefficients of the Cartan-Chern connection associated to the hermitian metric. In particular, then, (3.5) shows that in this case $T$ actually coincides with the torsion tensor of the connection.

But let us now return to equation (3.1) and holomorphic curvature. We shall say that a holomorphic curve $\varphi: U_{r} \rightarrow M$ realizes the holomorphic curvature at 0 if

$$
K\left(\varphi^{*} G\right)(0)=K_{F}\left(\varphi(0) ; \varphi^{\prime}(0)\right) .
$$

More generally, $\varphi$ realizes the holomorphic curvature at $\zeta_{0} \in U_{r}$ if $\varphi \circ \gamma_{\zeta_{0}}$ realizes it at 0 , where

$$
\gamma_{\zeta_{0}}(\zeta)=\frac{\zeta+\zeta_{0}}{1+\overline{\zeta_{0}} \zeta}
$$

is the unique automorphism of $U$ sending the origin to $\zeta_{0}$ with positive derivative at 0 . 
Proposition 3.2: Let $F$ be a strongly pseudoconvex smooth complex Finsler metric on a manifold $M$, and let $\varphi: U_{r} \rightarrow M$ be a holomorphic solution of (3.1). Then

(i) $\varphi$ realizes the holomorphic curvature at every point of $U_{r}$;

(ii) if $\left(\varphi(0) ; \varphi^{\prime}(0)\right) \in S^{1,0} M$, then $\varphi$ is a segment of infinitesimal complex geodesic for $F$.

Proof: (i) By Lemma 2.1 and (2.14), a holomorphic $\varphi: U_{r} \rightarrow M$ realizes the holomorphic curvature at 0 iff

$$
\eta^{\alpha}=-\lambda^{2} G^{\alpha \bar{\beta}}(p ; \xi) B_{\bar{\beta} ; i}(p ; \xi) \xi^{i}+c \xi^{\alpha}, \quad \alpha=1, \ldots, n,
$$

where $p=\varphi(0), v=\varphi^{\prime}(0)=\lambda \xi$ with $\xi \in S_{p}^{1,0} M, \eta=\varphi^{\prime \prime}(0)$ and $c \in \mathbb{C}$. Since, by (2.6), we have

$$
G^{\alpha \bar{\beta}} B_{\bar{\beta} ; i}=\Gamma_{; i}^{\alpha}-G_{; i} \xi^{\alpha}
$$

it follows that (3.7) is equivalent to

$$
\eta^{\alpha}=-\Gamma_{; i}^{\alpha}(p ; v) v^{i}+c_{1} v^{\alpha}, \quad \alpha=1, \ldots, n,
$$

with a possibly different $c_{1} \in \mathbb{C}$. But (3.8) with $c_{1}=0$ is just (3.1) evaluated in 0; so a holomorphic solution of (3.1) realizes the holomorphic curvature at the origin.

Now take $\zeta_{0} \in U_{r}$, and set $\psi=\varphi \circ \gamma_{\zeta_{0}}$. Then

$$
\begin{gathered}
\psi(0)=\varphi\left(\zeta_{0}\right) ; \\
\psi^{\prime}(0)=\left(1-\left|\zeta_{0}\right|^{2}\right) \varphi^{\prime}\left(\zeta_{0}\right) ; \\
\psi^{\prime \prime}(0)=\left(1-\left|\zeta_{0}\right|^{2}\right)^{2}\left(\varphi^{\prime \prime}\left(\zeta_{0}\right)+A\left(\zeta_{0}\right) \varphi^{\prime}\left(\zeta_{0}\right)\right) .
\end{gathered}
$$

So $\varphi$ realizes the holomorphic curvature at $\zeta_{0}$ iff

$$
\left(\varphi^{\prime \prime}\left(\zeta_{0}\right)+A\left(\zeta_{0}\right) \varphi^{\prime}\left(\zeta_{0}\right)\right)^{\alpha}=-\Gamma_{; i}^{\alpha}\left(\varphi\left(\zeta_{0}\right) ; \varphi^{\prime}\left(\zeta_{0}\right)\right)\left(\varphi^{\prime}\left(\zeta_{0}\right)\right)^{i}+c_{2}\left(\varphi^{\prime}\left(\zeta_{0}\right)\right)^{\alpha},
$$

and (i) follows.

(ii) Assume (3.1) holds. Then, recalling (2.6), we get

$$
\begin{aligned}
G_{\alpha}\left(\varphi ; \varphi^{\prime}\right)\left(\varphi^{\prime \prime}\right)^{\alpha}+A G\left(\varphi ; \varphi^{\prime}\right) & =G_{\alpha}\left(\varphi ; \varphi^{\prime}\right)\left[\left(\varphi^{\prime \prime}\right)^{\alpha}+A\left(\varphi^{\prime}\right)^{\alpha}\right] \\
& =-G_{\alpha}\left(\varphi ; \varphi^{\prime}\right) \Gamma_{; i}^{\alpha}\left(\varphi ; \varphi^{\prime}\right)\left(\varphi^{\prime}\right)^{i}=-G_{; i}\left(\varphi ; \varphi^{\prime}\right)\left(\varphi^{\prime}\right)^{i} .
\end{aligned}
$$

Therefore

$$
\frac{\partial}{\partial \zeta}\left[G\left(\varphi ; \varphi^{\prime}\right)\right]=G_{; i}\left(\varphi ; \varphi^{\prime}\right)\left(\varphi^{\prime}\right)^{i}+G_{\alpha}\left(\varphi ; \varphi^{\prime}\right)\left(\varphi^{\prime \prime}\right)^{\alpha}=-A G\left(\varphi ; \varphi^{\prime}\right) .
$$

Now, along the curve $t \mapsto e^{i \theta} t$ we have

$$
\frac{\partial}{\partial \zeta}=\frac{1}{2} e^{-i \theta} \frac{d}{d t}
$$

therefore $t \mapsto G\left(\varphi\left(e^{i \theta} t\right) ; \varphi^{\prime}\left(e^{i \theta} t\right)\right)$ is a solution of the Cauchy problem

$$
\left\{\begin{array}{l}
f^{\prime}(t)=\frac{4 t}{1-t^{2}} f(t), \\
f(0)=1
\end{array}\right.
$$

But $f(t)=\left(1-t^{2}\right)^{-2}$ is a solution of the same problem; therefore

$$
G\left(\varphi\left(e^{i \theta} t\right) ; \varphi^{\prime}\left(e^{i \theta} t\right)\right) \equiv \frac{1}{\left(1-t^{2}\right)^{2}}
$$

and we are done. 
So the main point now is to find when (3.1) has a holomorphic solution. Assume $\varphi: U_{r} \rightarrow M$ is such a solution, and apply $\partial / \partial \bar{\zeta}$ to (3.1). We get

$$
\frac{2}{\left(1-|\zeta|^{2}\right)^{2}}\left(\varphi^{\prime}\right)^{\alpha}=\Gamma_{; i \bar{\jmath}}^{\alpha}\left(\varphi ; \varphi^{\prime}\right)\left(\varphi^{\prime}\right)^{i} \overline{\left(\varphi^{\prime}\right)^{j}}+\Gamma_{\bar{\beta} ; i}^{\alpha}\left(\varphi ; \varphi^{\prime}\right)\left(\varphi^{\prime}\right)^{i} \overline{\left(\varphi^{\prime \prime}\right)^{\beta}}
$$

where, as before, $\Gamma_{; i \bar{\jmath}}^{\alpha}=\left(\Gamma_{; i}^{\alpha}\right)_{; \bar{j}}$ and $\Gamma_{\bar{\beta} ; i}^{\alpha}=\left(\Gamma_{; i}^{\alpha}\right)_{\bar{\beta}}$.

Now, (2.2) and (2.9) yield

$$
\Gamma_{\bar{\beta} ; i}^{\alpha} \overline{v^{\beta}}=G_{\bar{\beta}}^{\alpha \bar{\mu}} G_{\bar{\mu} ; i} \overline{v^{\beta}}+G^{\alpha \bar{\mu}} G_{\bar{\beta} \bar{\mu} ; i} \overline{v^{\beta}}=0 .
$$

So we can replace $\varphi^{\prime \prime}$ by $\varphi^{\prime \prime}+A \varphi^{\prime}$ in (3.9) and, calling in (3.1) again, we obtain

$$
\frac{2}{\left(1-|\zeta|^{2}\right)^{2}}\left(\varphi^{\prime}\right)^{\alpha}=\Gamma_{; i \bar{j}}^{\alpha}\left(\varphi ; \varphi^{\prime}\right)\left(\varphi^{\prime}\right)^{i} \overline{\left(\varphi^{\prime}\right)^{j}}-\Gamma_{\bar{\beta} ; i}^{\alpha}\left(\varphi ; \varphi^{\prime}\right) \Gamma_{; \bar{\jmath}}^{\bar{\beta}}\left(\varphi ; \varphi^{\prime}\right)\left(\varphi^{\prime}\right)^{i} \overline{\left(\varphi^{\prime}\right)^{j}}
$$

Now, if $\left(\varphi(0) ; \varphi^{\prime}(0)\right) \in S^{1,0} M$, then Proposition 3.2.(ii) yields

$$
G\left(\varphi(\zeta) ; \varphi^{\prime}(\zeta)\right)=\frac{1}{\left(1-|\zeta|^{2}\right)^{2}}
$$

therefore - setting $v=\varphi^{\prime}(\zeta)-(3.11)$ becomes

$$
\left[\Gamma_{; i \bar{\jmath}}^{\alpha}-\Gamma_{\bar{\beta} ; i}^{\alpha} \Gamma_{; \bar{\jmath}}^{\bar{\beta}}\right] v^{i} \overline{v^{j}}=2 G v^{\alpha}, \quad \alpha=1, \ldots, n
$$

So $(3.12)$ is a necessary condition for (3.1) to have a holomorphic solution. The interesting fact is that it is sufficient too:

Theorem 3.3: Let $F$ be a strongly pseudoconvex smooth complex Finsler metric on a manifold $M$. Then the Cauchy problem

$$
\left\{\begin{array}{l}
\left(\varphi^{\prime \prime}\right)^{\alpha}+A\left(\varphi^{\prime}\right)^{\alpha}=-\Gamma_{; i}^{\alpha}\left(\varphi ; \varphi^{\prime}\right)\left(\varphi^{\prime}\right)^{i} \quad \text { for } \alpha=1, \ldots, n \\
\varphi(0)=p, \quad \varphi^{\prime}(0)=v_{0}
\end{array}\right.
$$

admits a holomorphic solution for all $\left(p ; v_{0}\right) \in S^{1,0} M$ iff (3.12) holds. Furthermore, the solution, if exists, is unique.

Proof: We have already proved one direction; so assume (3.12) holds.

For any $e^{i \theta} \in \mathbf{S}^{1}$, let consider the Cauchy problem

$$
\left\{\begin{array}{l}
(\ddot{g}(t))^{\alpha}=-A(t)(\dot{g}(t))^{\alpha}-\Gamma_{; i}^{\alpha}(g(t) ; \dot{g}(t))(\dot{g}(t))^{i}, \quad \text { for } \alpha=1, \ldots, n, \\
g(0)=p, \quad \dot{g}(0)=e^{i \theta} v_{0} .
\end{array}\right.
$$

The standard ODE theory provides us with an $\varepsilon>0$ and uniquely determined maps $g_{e^{i \theta}}:(-\varepsilon, \varepsilon) \rightarrow M$ solving (3.14). Define $\varphi: U_{\varepsilon} \rightarrow M$ by

$$
\varphi(\zeta)=g_{\zeta /|\zeta|}(|\zeta|)
$$


and assume for a moment that $\varphi$ is holomorphic. Since, writing $\zeta=t e^{i \theta}$, we have

$$
\frac{\partial}{\partial \zeta}=-\frac{i e^{-i \theta}}{2 t}\left[\frac{\partial}{\partial \theta}+i t \frac{\partial}{\partial t}\right] \quad \text { and } \quad \frac{\partial}{\partial \bar{\zeta}}=\frac{i e^{i \theta}}{2 t}\left[\frac{\partial}{\partial \theta}-i t \frac{\partial}{\partial t}\right]
$$

it follows that

$$
\frac{\partial \varphi}{\partial \zeta}(\zeta)=\frac{\bar{\zeta}}{|\zeta|} \dot{g}_{\zeta /|\zeta|}(|\zeta|)
$$

and thus $\varphi$ is a holomorphic solution of (3.13). In conclusion, we must prove that, assuming (3.12), the map $\varphi$ defined by (3.15) is holomorphic. Note that, since a holomorphic map is uniquely determined by its restriction to the real axis, the uniqueness statement for (3.14) implies that $\varphi$ is the unique possible holomorphic solution of (3.13).

First of all, set $f_{0}(t)=\tanh t$ and

$$
\sigma_{\theta}(t)=g_{e^{i \theta}}(\tanh t)
$$

Then

$$
\dot{\sigma}_{\theta}=\left(f_{0}^{\prime}\right)\left(\dot{g}_{e^{i \theta}} \circ f_{0}\right) \quad \text { and } \quad \ddot{\sigma}_{\theta}=\left(f_{0}^{\prime}\right)^{2}\left[\left(\ddot{g}_{e^{i \theta}}+A \dot{g}_{e^{i \theta}}\right) \circ f_{0}\right] ;
$$

so $\sigma_{\theta}$ satisfies

$$
\left\{\begin{array}{l}
\ddot{\sigma}_{\theta}^{\alpha}=-\Gamma_{; i}^{\alpha}\left(\sigma_{\theta} ; \dot{\sigma}_{\theta}\right) \dot{\sigma}_{\theta}^{i}, \quad \text { for } \alpha=1, \ldots, n \\
\sigma_{\theta}(0)=p, \quad \dot{\sigma}_{\theta}(0)=e^{i \theta} v_{0} .
\end{array}\right.
$$

Set $h=G\left(\sigma_{\theta} ; \dot{\sigma}_{\theta}\right)$. Then

$$
\begin{aligned}
h^{\prime} & =G_{; i}\left(\sigma_{\theta} ; \dot{\sigma}_{\theta}\right) \dot{\sigma}_{\theta}^{i}+G_{\alpha}\left(\sigma_{\theta} ; \dot{\sigma}_{\theta}\right) \ddot{\sigma}_{\theta}^{\alpha} \\
& =G_{; i}\left(\sigma_{\theta} ; \dot{\sigma}_{\theta}\right) \dot{\sigma}_{\theta}^{i}+G_{\alpha \bar{\beta}}\left(\sigma_{\theta} ; \dot{\sigma}_{\theta}\right) \ddot{\sigma}_{\theta}^{\alpha} \overline{\dot{\sigma}_{\theta}^{\beta}} \\
& =G_{; i}\left(\sigma_{\theta} ; \dot{\sigma}_{\theta}\right) \dot{\sigma}_{\theta}^{i}-G_{\bar{\mu} ; i}\left(\sigma_{\theta} ; \dot{\sigma}_{\theta}\right) \dot{\sigma}_{\theta}^{i} \overline{\dot{\sigma}_{\theta}^{\mu}}=0 .
\end{aligned}
$$

So $h(t) \equiv h(0)=1$, and the curve $\sigma_{\theta}$ lifts to a curve $\tilde{\sigma}_{\theta}=d \sigma_{\theta}=\left(\sigma_{\theta} ; \dot{\sigma}_{\theta}\right)$ in $S^{1,0} M$.

Now, we define a global vector field $X \in \Gamma\left(T^{1,0}\left(S^{1,0} M\right)\right)$ by setting

$$
X_{\tilde{v}}=v^{i} \frac{\partial}{\partial z^{i}}-\Gamma_{; i}^{\alpha} v^{i} \frac{\partial}{\partial v^{\alpha}}
$$

where $\left(z^{1}, \ldots, z^{n} ; v^{1}, \ldots, v^{n}\right)$ are the local coordinates of $\tilde{v} \in\left(T^{1,0} M\right)_{0}$. It is not difficult to check that $X$ is globally defined, and that for $\tilde{v} \in S^{1,0} M$ it is actually true that $X_{\tilde{v}} \in T_{\tilde{v}}^{1,0}\left(S^{1,0} M\right)$, as claimed.

To proceed, we need to recall a basic fact of complex differential geometry. Let $N$ be a complex manifold, of complex dimension $m$. If we consider $N$ with its real structure, then $T N$ is a $(4 m)$-dimensional real vector bundle on $N$ endowed with a complex structure $J$. If we denote by $T^{c} N$ its complexification, then $T^{1,0} N$ is the $i$-eigenspace of $J$, and the canonical isomorphism $T^{1,0} N \rightarrow T N$ is given by

$$
Y \mapsto Y^{o}=Y+\bar{Y}
$$


where $\bar{Y}$ is the complex conjugate of $Y$ in $T^{c} N$. In particular, then,

$$
J Y^{o}=i(Y-\bar{Y})
$$

The aim of this observation is that, by construction, $\tilde{\sigma}_{\theta}$ is the integral curve in $S^{1,0} M$ of the vector field $X^{o}$ starting at $\left(p ; e^{i \theta} v_{0}\right)$. If we denote by $e^{t X^{o}}$ the local one-parameter group of diffeomorphisms induced by $X^{o}$ on $S^{1,0} M$, we may then write

$$
\tilde{\sigma}_{\theta}(t)=e^{t X^{o}}\left(e^{i \theta} \tilde{v}_{0}\right) \quad \text { and } \quad \sigma_{\theta}(t)=\pi\left(e^{t X^{o}} e^{i \theta} \tilde{v}_{0}\right)
$$

where $\pi: S^{1,0} M \rightarrow M$ is the canonical projection, and $\tilde{v}_{0}=\left(p ; v_{0}\right) \in S^{1,0} M$.

We need another vector field on $S^{1,0} M$. The map $\left(e^{i \theta},(p ; v)\right) \mapsto\left(p ; e^{i \theta} v\right)$ is a oneparameter group of diffeomorphisms of $S^{1,0} M$; therefore it is induced by a vector field $Z$, namely

$$
Z=i v^{\alpha} \frac{\partial}{\partial v^{\alpha}} \in \Gamma\left(T^{1,0}\left(S^{1,0} M\right)\right)
$$

note that $\pi_{*}(Z)=0$. Then $(3.19)$ becomes

$$
\tilde{\sigma}_{\theta}(t)=e^{t X^{o}} e^{\theta Z^{o}} \tilde{v}_{0} \quad \text { and } \quad \sigma_{\theta}(t)=\pi\left(e^{t X^{o}} e^{\theta Z^{o}} \tilde{v}_{0}\right)
$$

Now, we need to compute

$$
\left[X^{o}, J X^{o}\right]=i[X+\bar{X}, X-\bar{X}]=-2 i[X, \bar{X}]
$$

and

$$
\left[X^{o}, Z^{o}\right]=[X+\bar{X}, Z+\bar{Z}]=[X, Z]^{o}+[X, \bar{Z}]^{o} .
$$

Using local coordinates we find

$$
\begin{aligned}
{[X, Z] } & =-i \Gamma_{; j}^{\alpha} v^{j} \frac{\partial}{\partial v^{\alpha}}-i v^{\beta}\left[\frac{\partial}{\partial z^{\beta}}-\Gamma_{\beta ; j}^{\alpha} v^{j} \frac{\partial}{\partial v^{\alpha}}-\Gamma_{; \beta}^{\alpha} \frac{\partial}{\partial v^{\alpha}}\right] \\
& =-i\left[v^{\beta} \frac{\partial}{\partial z^{\beta}}-\Gamma_{; j}^{\alpha} v^{j} \frac{\partial}{\partial v^{\alpha}}\right]=-i X
\end{aligned}
$$

because, by $(2.2)$ and $(2.9), \Gamma_{\beta ; j}^{\alpha} v^{\beta}=\Gamma_{; j}^{\alpha}$. It is clear by the definitions and (3.10) that $[X, \bar{Z}]=0$; finally,

$$
\begin{aligned}
{[X, \bar{X}] } & =-\left[\Gamma_{; \bar{h} j}^{\bar{\alpha}}-\Gamma_{; j}^{\beta} \Gamma_{\beta ; \bar{h}}^{\bar{\alpha}}\right] v^{j} \overline{v^{h}} \frac{\partial}{\partial \bar{v}^{\alpha}}+\left[\Gamma_{; h \bar{\jmath}}^{\alpha}-\Gamma_{; \bar{\jmath}}^{\bar{\beta}} \Gamma_{\bar{\beta} ; h}^{\alpha}\right] \overline{v^{j}} v^{h} \frac{\partial}{\partial v^{\alpha}} \\
& =2\left(v^{\alpha} \frac{\partial}{\partial v^{\alpha}}-\overline{v^{\alpha}} \frac{\partial}{\partial \overline{v^{\alpha}}}\right)=-2 i Z^{o}
\end{aligned}
$$

where we used (3.12) on $S^{1,0} M$. So we get

$$
\left[X^{o}, J X^{o}\right]=-4 Z^{o} \quad \text { and } \quad\left[X^{o}, Z^{o}\right]=-J X^{o} .
$$


Now fix $\tau>0$, and set $\tilde{v}_{\tau}=e^{\tau X^{o}} \tilde{v}_{0}$. Put

$$
u(t)=e_{*}^{t X^{o}} Z_{e^{-t X^{o}} \tilde{v}_{\tau}}^{o} \in T_{\tilde{v}_{\tau}}\left(S^{1,0} M\right) .
$$

Then

$$
\frac{d u}{d t}(t)=\frac{d}{d t}\left(e_{*}^{t X^{o}} Z_{e^{-t X^{o}} \tilde{v}_{\tau}}^{o}\right)=-e_{*}^{t X^{o}}\left\{\mathcal{L}_{X^{o}} Z^{o}\right\}_{e^{-t X^{o}} \tilde{v}_{\tau}}=e_{*}^{t X^{o}}\left(J X^{o}\right)_{e^{-t X^{o}} \tilde{v}_{\tau}}
$$

where $\mathcal{L}_{X^{\circ}}$ is the Lie derivative, and

$$
\frac{d^{2} u}{d t^{2}}(t)=\frac{d}{d t}\left(e_{*}^{t X^{o}}\left(J X^{o}\right)_{e^{-t X^{o}} \tilde{v}_{\tau}}\right)=-e_{*}^{t X^{o}}\left\{\mathcal{L}_{X^{o}}\left(J X^{o}\right)\right\}_{e^{-t X^{o}} \tilde{v}_{\tau}}=4 e_{*}^{t X^{o}} Z_{e^{-t X^{o}} \tilde{v}_{\tau}} .
$$

In other words, $u(t)$ is a solution of the Cauchy problem

$$
\left\{\begin{array}{l}
\ddot{u}=4 u \\
u(0)=Z_{\tilde{v}_{\tau}}^{o}, \quad \dot{u}(0)=\left(J X^{o}\right)_{\tilde{v}_{\tau}} .
\end{array}\right.
$$

Therefore

$$
u(t)=\frac{1}{4} e^{2 t}\left(2 Z_{\tilde{v}_{\tau}}^{o}+\left(J X^{o}\right)_{\tilde{v}_{\tau}}\right)+\frac{1}{4} e^{-2 t}\left(2 Z_{\tilde{v}_{\tau}}^{o}-\left(J X^{o}\right)_{\tilde{v}_{\tau}}\right),
$$

and, in particular,

$$
\pi_{*} e_{*}^{\tau X^{o}} Z_{\tilde{v}_{0}}^{o}=\pi_{*} u(\tau)=\frac{e^{2 \tau}-e^{-2 \tau}}{4} \pi_{*}\left(J X^{o}\right)_{\tilde{v}_{\tau}}=\frac{e^{2 \tau}-e^{-2 \tau}}{4} J \pi_{*} X_{\tilde{v}_{\tau}}^{o} .
$$

We are almost done. Recalling (3.15), (3.16), (3.17) and (3.20), it is clear that we should prove that

$$
\left.\frac{\partial}{\partial \theta} \pi\left(e^{(\operatorname{atanh} t) X^{o}} e^{\theta Z^{o}} \tilde{v}_{0}\right)\right|_{\theta=0}=\left.t J \frac{\partial}{\partial t} \pi\left(e^{(\operatorname{atanh} t) X^{o}} e^{\theta Z^{o}} \tilde{v}_{0}\right)\right|_{\theta=0}
$$

where we may take $\theta=0$ because $\tilde{v}_{0}$ is generic. Let us compute; using (3.22) we get

$$
\left.\frac{\partial}{\partial \theta} \pi\left(e^{(\operatorname{atanh} t) X^{o}} e^{\theta Z^{o}} \tilde{v}_{0}\right)\right|_{\theta=0}=\pi_{*} e_{*}^{(\operatorname{atanh} t) X^{o}} Z_{\tilde{v}_{0}}^{o}=\frac{t}{1-t^{2}} J \pi_{*} X_{\tilde{v}_{\operatorname{atanh} t}}^{o}
$$

whereas

$$
\left.\frac{\partial}{\partial t} \pi\left(e^{(\operatorname{atanh} t) X^{o}} e^{\theta Z^{o}} \tilde{v}_{0}\right)\right|_{\theta=0}=\frac{\partial}{\partial t} \pi\left(e^{(\operatorname{atanh} t) X^{o}} \tilde{v}_{0}\right)=\frac{1}{1-t^{2}} \pi_{*} X_{\tilde{v}_{\operatorname{atanh} t}^{o}},
$$

and the proof is complete.

So we have found a necessary and sufficient condition for the existence of segments of geodesic complex curves: 
Corollary 3.4: Let $F$ be a strongly pseudoconvex smooth complex Finsler metric on a manifold $M$. Then:

(i) if (3.12) holds, then for any $(p ; \xi) \in S^{1,0} M$ there is a segment of infinitesimal complex geodesic tangent to $(p ; \xi)$;

(ii) there exists a (unique) segment of geodesic complex curve tangent to $(p$; $\xi$ ) for any $(p ; \xi) \in S^{1,0} M$ iff $F$ is Kähler and (3.12) holds.

Proof: (i) Theorem 3.3 and Proposition 3.2.(ii).

(ii) In $[\mathrm{AP}]$ it is shown that a segment of geodesic complex curve is a holomorphic solution of the system (3.1)-(3.2). The assertion then follows from Theorem 3.3 and Proposition 3.1.

A natural question now is whether the completeness of the metric $F$ - together with Kähler and (3.12) — would imply the existence of geodesic complex curves defined on the whole unit disk $U$. The answer is positive, but for the proof we beforehand need a discussion of the geometrical meaning of (3.12).

Thanks to Proposition 3.2.(i), we know that a holomorphic solution of (3.1) realizes the holomorphic curvature and it is an isometry from the Poincaré metric to $F$; in particular, thus, the holomorphic curvature along the curve should be -4 . This suggests to look for a connection between (3.12) and the holomorphic curvature; and indeed the next result shows that the connection is provided by a sort of simmetry condition on the curvature. Analogously to the tensor $T_{\alpha i \bar{\mu}}$ previously introduced, set

$$
\begin{aligned}
H_{\alpha i \bar{\mu} \bar{\jmath}} & =G_{\tau \bar{\mu}}\left(\Gamma_{i ; \alpha}^{\tau}-\Gamma_{\alpha ; i}^{\tau}\right)_{; \bar{\jmath}}+G_{\tau \bar{\mu} i} \Gamma_{; \alpha \bar{\jmath}}^{\tau}-G_{\tau \bar{\mu} \alpha} \Gamma_{; i \bar{\jmath}}^{\tau} \\
& =\left(G_{\tau \bar{\mu}} \Gamma_{; \alpha \bar{\jmath}}^{\tau}\right)_{i}-\left(G_{\tau \bar{\mu}} \Gamma_{; i \bar{\jmath}}^{\tau}\right)_{\alpha} ;
\end{aligned}
$$

it is a $(4,0)$-tensor on $\left(T^{1,0} M\right)_{0}$. Note that

$$
H_{\alpha i \bar{\mu} \bar{\jmath}} \overline{v^{\mu}} v^{i} \overline{v^{j}}=\left[G_{\tau}\left(\Gamma_{i ; \alpha}^{\tau}-\Gamma_{\alpha ; i}^{\tau}\right)_{; \bar{\jmath}}-G_{\tau \alpha} \Gamma_{; i \bar{\jmath}}^{\tau}\right] v^{i} \overline{v^{j}}
$$

Then

Theorem 3.5: Let $F$ be a strongly pseudoconvex smooth complex Finsler metric on a manifold $M$. Then (3.12) holds iff $K_{F} \equiv-4$ and

$$
H_{\alpha i \bar{\mu} \bar{\jmath}} \overline{v^{\mu}} v^{i} \overline{v^{j}}=0, \quad \alpha=1, \ldots, n
$$

Proof: We start by showing that (3.12) implies $K_{F} \equiv-4$. Indeed, take $(p ; \xi) \in S^{1,0} M$. Then, recalling (2.2), (2.6), and (2.10), we get

$$
G_{\alpha} \Gamma_{\bar{\beta} ; i}^{\alpha}=G_{\alpha} G_{\bar{\beta}}^{\alpha \bar{\mu}} G_{\bar{\mu} ; i}+G_{\alpha} G^{\alpha \bar{\mu}} G_{\bar{\mu} \bar{\beta} ; i}=0
$$

Therefore (3.12) yields

$$
K_{F}(p ; \xi)=-2 G_{\alpha} \Gamma_{; i \bar{\jmath}}^{\alpha} \xi^{i} \overline{\xi^{j}}=-4 G_{\alpha} \xi^{\alpha}-G_{\alpha} \Gamma_{\bar{\beta} ; i}^{\alpha} \Gamma_{\bar{\jmath}}^{\bar{\beta}} \xi^{i} \overline{\xi^{j}}=-4 G_{\alpha} \xi^{\alpha}=-4
$$


From now on we shall assume $K_{F} \equiv-4$; we ought to prove that in this case (3.12) is equivalent to (3.24), that is, by $(3.23)$, to

$$
G_{\tau}\left(\Gamma_{i ; \alpha}^{\tau}-\Gamma_{\alpha ; i}^{\tau}\right)_{;} v^{i} \overline{v^{j}}=G_{\tau \alpha} \Gamma_{; i \bar{\jmath}}^{\tau} v^{i} \overline{v^{j}}, \quad \alpha=1, \ldots, n .
$$

By $(2.18), K_{F} \equiv-4$ is equivalent to

$$
G_{\beta} \Gamma_{; i \bar{\jmath}}^{\beta} v^{i \overline{v^{j}}}=2 G^{2} .
$$

Differentiating with respect to $\overline{v^{\nu}}$ we get

$$
4 G G_{\bar{\nu}}=\left[G_{\beta \bar{\nu}} \Gamma_{; i \bar{\nu}}^{\beta}+G_{\beta} \Gamma_{\bar{\nu} ; i \bar{\jmath}}^{\beta}\right] v^{i} v^{j}+G_{\beta} \Gamma_{; i \bar{\nu}}^{\beta} v^{i}
$$

multiplying by $G^{\alpha \bar{\nu}}$, and recalling (2.6), we obtain

$$
4 G v^{\alpha}=\left[\Gamma_{; i \bar{\jmath}}^{\alpha}+G^{\alpha \bar{\nu}} G_{\beta} \Gamma_{\bar{\nu} ; i \bar{j}}^{\beta}\right] v^{i} \bar{v}^{\bar{j}}+G^{\alpha \bar{\nu}} G_{\beta} \Gamma_{; i \bar{\nu}}^{\beta} v^{i} .
$$

Now,

$$
\begin{gathered}
\Gamma_{; i \bar{\jmath}}^{\beta}=G^{\beta \bar{\mu}} G_{\bar{\mu} ; i \bar{\jmath}}-G^{\beta \bar{\tau}} G_{\sigma \bar{\tau} ; \bar{j}} \Gamma_{; i}^{\sigma} ; \\
\Gamma_{\bar{\nu} ; i \bar{\jmath}}^{\beta}=G_{\bar{\nu}}^{\beta \bar{\mu}} G_{\bar{\mu} ; i \bar{\jmath}}+G^{\beta \bar{\mu}} G_{\bar{\mu} \bar{\nu} ; i \bar{\jmath}}-G_{\bar{\nu}}^{\beta \bar{\tau}} G_{\sigma \bar{\tau} ; \bar{j}} \Gamma_{; i}^{\sigma}-G^{\beta \bar{\tau}} G_{\sigma \bar{\tau} \bar{\nu} ; \bar{j}} \Gamma_{; i}^{\sigma}-G^{\beta \bar{\tau}} G_{\sigma \bar{\tau} ; \bar{j}} \Gamma_{\bar{\nu} ; i}^{\sigma} .
\end{gathered}
$$

Therefore, using (2.2), (2.3), (2.4), (2.6), (2.8) and (2.10), we get

$$
\begin{aligned}
G^{\alpha \bar{\nu}} G_{\beta} \Gamma_{\bar{\nu} ; i \bar{\jmath}}^{\beta} & =-G^{\alpha \bar{\nu}} G_{\sigma ; \bar{j}} \Gamma_{\bar{\nu} ; i}^{\sigma}=-G^{\alpha \bar{\nu}} G_{\sigma ; \bar{j}}\left(G_{\bar{\nu}}^{\sigma \bar{\mu}} G_{\bar{\mu} ; i}+G^{\sigma \bar{\mu}} G_{\bar{\mu} \bar{\nu} ; i}\right) \\
& =-\Gamma_{; \bar{j}}^{\bar{\mu}} G^{\alpha \bar{\nu}} G_{\bar{\mu} \bar{\nu} ; i}+\Gamma_{; \bar{\jmath}}^{\bar{\gamma}} G^{\alpha \bar{\nu}} G^{\delta \bar{\mu}} G_{\delta \bar{\gamma} \bar{\nu}} G_{\bar{\mu} ; i} \\
& =-\Gamma_{; \bar{j}}^{\mu}\left(G^{\alpha \bar{\nu}} G_{\bar{\mu} \bar{\nu} ; i}+G_{\bar{\mu}}^{\alpha \bar{\nu}} G_{\bar{\nu} ; i}\right)=-\Gamma_{\bar{\mu} ; i}^{\alpha} \Gamma_{\bar{\jmath}}^{\bar{\mu}} .
\end{aligned}
$$

So (3.26) becomes

$$
4 G v^{\alpha}=\left[\Gamma_{; i \bar{\jmath}}^{\alpha}-\Gamma_{\bar{\mu} ; i}^{\alpha} \Gamma_{; \bar{j}}^{\bar{\mu}}\right] v^{i} \overline{v^{j}}+G^{\alpha \bar{\nu}} G_{\beta} \Gamma_{; i \bar{\nu}}^{\beta} v^{i} .
$$

Now, in (2.16) we showed that

$$
G^{\alpha \bar{\nu}} G_{\beta} \Gamma_{; i \bar{\nu}}^{\beta} v^{i}=G^{\alpha \bar{\nu}}\left[G_{; i \bar{\nu}}-\Gamma_{; j}^{\bar{\tau}} G_{\bar{\tau} ; i}\right] v^{i} .
$$

Since, by (2.4) and (2.9),

$$
\Gamma_{\bar{\jmath} ; \bar{\nu}}^{\bar{\tau}} \overline{v^{j}}=G^{\sigma \bar{\tau}} G_{\sigma \bar{\jmath} ; \bar{\nu}} \overline{v^{j}}=\Gamma_{; \bar{\nu}}^{\bar{\tau}},
$$

we get

$$
\begin{aligned}
G^{\alpha \bar{\nu}} G_{\beta} \Gamma_{; i \bar{\nu}}^{\beta} v^{i} & =G^{\alpha \bar{\nu}}\left[G_{\bar{j} ; i \bar{\nu}}-G_{\bar{\tau} ; i} \Gamma_{\bar{\jmath} ; \bar{\nu}}^{\bar{\tau}}\right] v^{i \overline{v^{j}}} \\
& =G^{\alpha \bar{\nu}}\left[G_{\bar{\nu} ; i \bar{\jmath}}-G_{\bar{\tau} ; i} \Gamma_{\bar{\nu} ; \bar{j}}^{\bar{\tau}}\right] v^{i} v^{\bar{j}}+G^{\alpha \bar{\nu}}\left[\left(G_{\bar{\jmath} ; \bar{\nu}}-G_{\bar{\nu} ; \bar{\jmath}}\right)_{; i}-G_{\bar{\tau} ; i}\left(\Gamma_{\bar{\jmath} ; \bar{\nu}}^{\overline{\bar{\tau}}}-\Gamma_{\bar{\nu} ; \bar{\jmath}}^{\overline{\bar{\nu}}}\right)\right] v^{i} \overline{v^{j}} .
\end{aligned}
$$


Now (2.8) yields

$$
\begin{aligned}
G^{\alpha \bar{\nu}} G_{\bar{\tau} ; i} \Gamma_{\bar{\nu} ; \bar{j}}^{\bar{\tau}} & =G^{\alpha \bar{\nu}} G_{\sigma \bar{\nu} ; \bar{\jmath}} \Gamma_{; i}^{\sigma}-G^{\alpha \bar{\nu}} G_{\delta \bar{\mu} \bar{\nu}} \Gamma_{; j \bar{j}}^{\bar{\mu}} \Gamma_{; i}^{\delta} \\
& =G^{\alpha \bar{\nu}} G_{\sigma \bar{\nu} ; \bar{\jmath}} \Gamma_{; i}^{\sigma}+\Gamma_{\bar{\mu} ; i}^{\alpha} \Gamma_{; \bar{\jmath}}^{\bar{\mu}}-G^{\alpha \bar{\nu}} G_{\bar{\mu} \bar{\nu} ; i} \Gamma_{; \bar{\jmath}}^{\bar{\mu}} ;
\end{aligned}
$$

so, by (3.27),

$$
G^{\alpha \bar{\nu}}\left(G_{\bar{\nu} ; i \bar{\jmath}}-G_{\bar{\tau} ; i} \Gamma_{\bar{\nu} ; \bar{\jmath}}^{\bar{\tau}}\right)=\left(\Gamma_{; i \bar{\jmath}}^{\alpha}-\Gamma_{\bar{\mu} ; i}^{\alpha} \Gamma_{; \bar{j}}^{\bar{\mu}}\right)+G^{\alpha \bar{\nu}} G_{\bar{\mu} \bar{\nu} ; i} \Gamma_{; \bar{\jmath}}^{\bar{\mu}} .
$$

Summing up, we have found

$$
\begin{aligned}
G^{\alpha \bar{\nu}} G_{\beta} \Gamma_{; i \bar{\nu}}^{\beta} v^{i}=[ & \left.\Gamma_{; i \bar{\jmath}}^{\alpha}-\Gamma_{\bar{\mu} ; i}^{\alpha} \Gamma_{; \bar{\jmath}}^{\bar{\mu}}\right] v^{i \overline{v^{j}}} \\
& +G^{\alpha \bar{\nu}}\left[G_{\bar{\tau} \bar{\nu} ; i} \Gamma_{; \bar{\jmath}}^{\bar{\tau}}+\left(G_{\bar{\jmath} ; \bar{\nu}}-G_{\bar{\nu} ; \bar{\jmath}}\right)_{; i}-G_{\bar{\tau} ; i}\left(\Gamma_{\bar{\jmath} ; \bar{\nu}}^{\bar{\tau}}-\Gamma_{\bar{\nu} ; \bar{\jmath}}^{\bar{\tau}}\right)\right] v^{i} v^{j} .
\end{aligned}
$$

For the moment, set

$$
T_{\bar{\nu}}=\left[G_{\bar{\jmath} ; \bar{\nu}}-G_{\bar{\nu} ; \bar{\jmath}}+G_{\bar{\tau} \bar{\nu}} \Gamma_{; \bar{\jmath}}^{\bar{\tau}}\right] \overline{v^{j}}=T_{\bar{\nu} \bar{\jmath} \mu} v^{\mu} \overline{v^{j}}
$$

recall that (3.6) says that

$$
T_{\bar{\nu}}=G_{\bar{\tau}}\left(\Gamma_{\bar{\jmath} ; \bar{\nu}}^{\bar{\tau}}-\Gamma_{\bar{\nu} ; \bar{\jmath}}^{\bar{\tau}}\right) \overline{v^{j}}
$$

Therefore

$$
\begin{gathered}
\left(G_{\bar{\jmath} ; \bar{\nu}}-G_{\bar{\nu} ; \bar{\jmath}}\right)_{; i} \overline{v^{j}}=\left(T_{\bar{\nu}}\right)_{; i}-\left(G_{\bar{\tau} \bar{\nu}} \Gamma_{; \bar{\jmath}}^{\bar{\tau}}\right)_{; i} \overline{v^{j}}=\left(T_{\bar{\nu}}\right)_{; i}-\left[G_{\bar{\tau} \bar{\nu} ; i} \Gamma_{; \bar{\jmath}}^{\bar{\tau}}+G_{\bar{\tau} \bar{\nu}} \Gamma_{; j i}^{\bar{\tau}}\right] \overline{v^{j}} \\
G_{\bar{\tau} ; i}\left(\Gamma_{\bar{\jmath} ; \bar{\nu}}^{\bar{\tau}}-\Gamma_{\bar{\nu} ; \bar{\jmath}}^{\bar{\tau}}\right) \overline{v^{j}}=\left(T_{\bar{\nu}}\right)_{; i}-G_{\bar{\tau}}\left(\Gamma_{\bar{\jmath} ; \bar{\nu}}^{\bar{\tau}}-\Gamma_{\bar{\nu} ; \bar{\jmath}}^{\bar{\tau}}\right)_{; i} \overline{v^{j}}
\end{gathered}
$$

In conclusion, we have shown that

$$
G^{\alpha \bar{\nu}} G_{\beta} \Gamma_{; i \bar{\nu}}^{\beta} v^{i}=\left[\Gamma_{; i \bar{j}}^{\alpha}-\Gamma_{\bar{\mu} ; i}^{\alpha} \Gamma_{; \bar{\jmath}}^{\bar{\mu}}\right] v^{i} v^{\bar{j}}+G^{\alpha \bar{\nu}}\left[G_{\bar{\tau}}\left(\Gamma_{\bar{\jmath} ; \bar{\nu}}^{\bar{\tau}}-\Gamma_{\bar{\nu} ; \bar{\jmath}}^{\bar{\tau}}\right)_{; i}-G_{\bar{\nu} \bar{\tau}} \Gamma_{; \bar{j} i}^{\bar{\tau}}\right] v^{i} v^{\bar{j}} .
$$

Recalling (3.28), we have obtained

$$
4 G v^{\alpha}=2\left[\Gamma_{; i \bar{j}}^{\alpha}-\Gamma_{\bar{\mu} ; i}^{\alpha} \Gamma_{; \bar{j}}^{\bar{\mu}}\right] v^{i} \overline{v^{j}}+G^{\alpha \bar{\nu}}\left[G_{\bar{\tau}}\left(\Gamma_{\bar{j} ; \bar{\nu}}^{\bar{\tau}}-\Gamma_{\bar{\nu} ; \bar{j}}^{\bar{\tau}}\right)_{; i}-G_{\bar{\nu} \bar{\tau}} \Gamma_{; \bar{j} i}^{\bar{\tau}}\right] v^{i} \overline{v^{j}}
$$

and the assertion follows.

If $G(p ; v)=g_{\mu \bar{\nu}}(p) v^{\mu} \overline{v^{\nu}}$ is an hermitian metric, then the tensor $H_{\alpha i \bar{\mu} \bar{\jmath}}$ becomes

$$
H_{\alpha i \bar{\mu} \bar{\jmath}}=g_{\tau \bar{\mu}} \frac{\partial}{\partial \overline{z^{j}}} T_{i \alpha}^{\tau}=R_{i \alpha \bar{\mu} \bar{\jmath}}-R_{\alpha i \bar{\mu} \bar{\jmath}}
$$

where $T_{i \alpha}^{\tau}$ is the torsion of the Chern connection associated to the hermitian metric, and $R_{\alpha i \bar{\mu} \bar{\jmath}}$ is the Riemannian curvature tensor of the connection. So (3.24) is equivalent to

$$
R_{i \alpha \bar{\mu} \bar{\jmath}} v^{i} \overline{v^{\mu}} \overline{v^{j}}=R_{\alpha i \bar{\mu} \bar{\jmath}} v^{i} \overline{v^{\mu}} \overline{v^{j}}
$$

for all $v \in T^{1,0} M$. So (3.24) may be interpreted as a simmetry condition on a curvature tensor; more precisely, as anticipated in the introduction, a simmetry condition on the Chern connection induced by the Finsler metric on the vertical subbundle of $T^{1,0}\left(T^{1,0} M\right)$. Finally, we also remark that — at least in the hermitian case - (3.24) in particular holds when $\bar{\partial} T \equiv 0$.

Now that we have an idea of the geometrical meaning of (3.12), we may return to the study of geodesic complex curves. As anticipated, we are now able to prove the following 
Theorem 3.6: Let $F$ be a strongly pseudoconvex smooth complete complex Finsler metric on a manifold $M$. Assume that the holomorphic curvature of $F$ is identically -4 and that (3.24) holds. Then for every $(p ; \xi) \in S^{1,0} M$ there is a unique holomorphic solution $\varphi: U \rightarrow M$ of (3.1) defined on the whole unit disk $U$ such that $\varphi(0)=p$ and $\varphi^{\prime}(0)=\xi$.

Proof: First of all, we remark that the distribution $\mathbb{C} X^{o} \oplus \mathbb{C} Z^{o} \subset T\left(S^{1,0} M\right)$ is involutive. Indeed, Theorem 3.5 shows that (3.12) holds, and in the proof of Theorem 3.3 we have already computed $\left[X^{o}, J X^{o}\right]=-4 Z^{o}$ and $\left[X^{o}, Z^{o}\right]=-J X^{o}$. For the remaining brackets, using (3.21) we get

$$
\left[X^{o}, J Z^{o}\right]=X^{o}, \quad\left[J X^{o}, Z^{o}\right]=X^{o}, \quad\left[J X^{o}, J Z^{o}\right]=J X^{o}, \quad\left[Z^{o}, J Z^{o}\right]=0 .
$$

Let $\widetilde{N}$ denote the integral leaf of this distribution passing through $(p ; \xi)$. From the proof of Theorem 3.3 it follows that $N=\pi(\tilde{N}) \subset M$ is a Riemann surface locally parametrized by the holomorphic solutions of (3.1). In particular, $F$ restricted to $N$ is a complete hermitian metric of constant Gaussian curvature -4 , because of Proposition 3.2. Thus there is a unique holomorphic covering map $\psi: U \rightarrow N$ which is an isometry between the Poincaré metric on $U$ and $F$ restricted to $N$ and such that $\psi(0)=p$ and $\psi^{\prime}(0)=\xi$. But if $\varphi: U_{\varepsilon} \rightarrow N$ is the holomorphic solution of (3.1) with $\varphi(0)=p$ and $\varphi^{\prime}(0)=\xi$, then $\varphi$ too is an isometry between the Poincaré metric restricted to $U_{\varepsilon}$ and $F$ restricted to $N$; it follows that $\varphi=\left.\psi\right|_{U_{\varepsilon}}$, and $\psi$ is the extension of $\varphi$ to the whole $U$ we were looking for.

Corollary 3.7: Let $F$ be a strongly pseudoconvex smooth complete complex Finsler metric on a manifold $M$. Assume that the holomorphic curvature of $F$ is identically -4 and that (3.24) holds. Then:

(i) for any $(p ; \xi) \in S^{1,0} M$ there is an infinitesimal complex geodesic tangent to $(p ; \xi)$;

(ii) if moreover $F$ is Kähler, then for any $(p ; \xi) \in S^{1,0} M$ there is a unique geodesic complex curve tangent to $(p ; \xi)$.

Proof: Theorems 3.5, 3.6 and Propositions 3.1 and 3.2.

Actually, we can even get a sort of punctual version of the latter result. As usual, we need a computation, which by the way clarifies the relationship among (3.12) and the two torsion tensors we introduced, $T$ and $H$. For the sake of simplicity, set

$$
\Sigma_{; i \bar{\jmath}}^{\alpha}=\Gamma_{; i \bar{\jmath}}^{\alpha}-\Gamma_{\bar{\mu} ; i}^{\alpha} \Gamma_{; \bar{j}}^{\bar{\mu}}
$$

In particular, (3.12) becomes $\Sigma_{; i \bar{\jmath}}^{\alpha} v^{i} v^{j}=2 G v^{\alpha}$.

Proposition 3.8: Let $F$ be a strongly pseudoconvex smooth complex Finsler metric on a manifold $M$. Then

$$
H_{\alpha i \bar{\mu} \bar{\jmath}} \overline{v^{\mu}} \overline{v^{j}}=\bar{X}\left(T_{\alpha i \bar{\mu}} \overline{v^{\mu}}\right)+\left(G_{i \sigma} \Sigma_{; \alpha \bar{\jmath}}^{\sigma}-G_{\alpha \sigma} \Sigma_{; i \bar{\jmath}}^{\sigma}\right) \overline{v^{j}},
$$

for all $i, \alpha=1, \ldots, n$, where $\bar{X}$ is the complex conjugate of the vector field defined in (3.18). In particular,

$$
H_{\alpha i \bar{\mu} \bar{\jmath}} \overline{v^{\mu}} v^{i} \overline{v^{j}}=\bar{X}\left(T_{\alpha i \bar{\mu}} \overline{v^{\mu}} v^{i}\right)-G_{\alpha \sigma} \sum_{; i \bar{\jmath}}^{\sigma} v^{i} \overline{v^{j}}, \quad \alpha=1, \ldots, n .
$$


Proof: By (3.5) and (3.18)

$$
\begin{gathered}
T_{\alpha i \bar{\mu}} \overline{v^{\mu}}=G_{\beta}\left(\Gamma_{i ; \alpha}^{\beta}-\Gamma_{\alpha ; i}^{\beta}\right), \\
\bar{X}=\overline{v^{j}} \frac{\partial}{\partial \overline{z^{j}}}-\Gamma_{; \bar{j}}^{\bar{\gamma}} \overline{v^{j}} \frac{\partial}{\partial \overline{v^{\gamma}}} .
\end{gathered}
$$

Thus

$$
\begin{aligned}
\bar{X}\left(T_{\alpha i \bar{\mu}} \overline{v^{\mu}}\right)=G_{\beta}\left[\left(\Gamma_{i ; \alpha}^{\beta}-\Gamma_{\alpha ; i}^{\beta}\right)_{; \bar{j}}-\Gamma_{; \bar{j}}^{\bar{\gamma}}\left(\Gamma_{i ; \alpha}^{\beta}-\Gamma_{\alpha ; i}^{\beta}\right)_{\bar{\gamma}}\right] \overline{v^{j}} \\
+\left[G_{\beta ; \bar{j}}-G_{\beta \bar{\gamma}} \Gamma_{; j}^{\bar{\gamma}}\right]\left(\Gamma_{i ; \alpha}^{\beta}-\Gamma_{\alpha ; i}^{\beta}\right) \overline{v^{j}} .
\end{aligned}
$$

Now, $G_{\beta \bar{\gamma}} \Gamma_{; \bar{j}}^{\bar{\gamma}}=G_{\beta ; \bar{j}}$, and so the second addendum in (3.30) vanishes. Next, recalling (3.4) and the usual formulas,

$$
G_{\beta}\left(\Gamma_{i ; \alpha}^{\beta}-\Gamma_{\alpha ; i}^{\beta}\right)_{\bar{\gamma}}=-\left[G_{i \sigma} \Gamma_{\bar{\gamma} ; \alpha}^{\sigma}-G_{\alpha \sigma} \Gamma_{\bar{\gamma} ; i}^{\sigma}\right] .
$$

Therefore

$$
\begin{aligned}
\bar{X}\left(T_{\alpha i \bar{\mu}} \overline{v^{\mu}}\right) & =G_{\beta}\left(\Gamma_{i ; \alpha}^{\beta}-\Gamma_{\alpha ; i}^{\beta}\right)_{; \bar{\jmath}} \overline{v^{j}}+\left[G_{i \sigma} \Gamma_{\bar{\gamma} ; \alpha}^{\sigma} \Gamma_{; \bar{\jmath}}^{\bar{\gamma}}-G_{\alpha \sigma} \Gamma_{\bar{\gamma} ; i}^{\sigma} \Gamma_{; j}^{\bar{\gamma}}\right] \overline{v^{j}} \\
& =G_{\beta}\left(\Gamma_{i ; \alpha}^{\beta}-\Gamma_{\alpha ; i}^{\beta}\right)_{; \bar{\jmath}} \overline{v^{j}}-\left[G_{i \sigma} \Sigma_{; \alpha \bar{\jmath}}^{\sigma}-G_{\alpha \sigma} \Sigma_{; i \bar{\jmath}}^{\sigma} \overline{v^{j}}+\left(G_{i \sigma} \Gamma_{; \alpha \bar{\jmath}}^{\sigma}-G_{\alpha \sigma} \Gamma_{; i \bar{\jmath}}^{\sigma}\right) \overline{v^{j}}\right. \\
& =H_{\alpha i \bar{\mu} \bar{\jmath}} \overline{v^{\mu}} \overline{v^{j}}-\left[G_{i \sigma} \Sigma_{; \alpha \bar{\jmath}}^{\sigma}-G_{\alpha \sigma} \Sigma_{; i \bar{j}}^{\sigma}\right]
\end{aligned}
$$

and the assertion follows.

As a corollary we have

Corollary 3.9: Let $F$ be a strongly pseudoconvex smooth complex Finsler metric on a manifold $M$. Assume that the holomorphic curvature of $F$ is identically -4 and that (3.24) holds. Take $\left(p_{0} ; \xi_{0}\right) \in S^{1,0} M$. Then there is a segment of geodesic complex curve tangent to $\left(p_{0} ; \xi_{0}\right)$ iff $F$ is Kähler at $\left(p_{0} ; \xi_{0}\right)$, that is iff

$$
T_{\alpha i \bar{\mu}}\left(p_{0} ; \xi_{0}\right) \xi_{0}^{i} \overline{\xi_{0}^{\mu}}=0, \quad \alpha=1, \ldots, n .
$$

The segment, if exists, is unique. Furthermore, if $F$ is complete then the segment of geodesic complex curve actually extends to a whole geodesic complex curve.

Proof: One direction is known. Conversely, assume (3.31) holds. By Theorem 3.5,

$$
G_{\alpha \sigma} \Sigma_{; i \bar{\jmath}}^{\sigma} v^{i} \overline{v^{j}}=0 ;
$$

hence, by Proposition 3.8, $X\left(T_{\alpha i \bar{\mu}} v^{i} \overline{v^{\mu}}\right)=0$. So $T_{\alpha i \bar{\mu}} v^{i}{\overline{v^{\mu}}}$ is constant (and thus zero) along the solution of (3.1) tangent to $\left(p_{0} ; \xi_{0}\right)$; the assertion then follows from Proposition 3.1.

As already discussed in the introduction, one of the motivations behind this work was to find a differential description of the properties of the Kobayashi metric in strongly convex domains. We conclude then with the following:

Corollary 3.10: Let $F$ be a strongly pseudoconvex smooth complete complex Finsler metric on a manifold $M$. Assume that (3.12) holds or, equivalently, that $K_{F} \equiv-4$ and (3.24) holds. Then $F$ is the Kobayashi metric of $M$.

Proof: By Theorem 3.5, in both cases the holomorphic curvature of $F$ is -4 . Furthermore, by Theorem 3.6 F is realizable. The assertion then follows from Proposition 1.7. 


\section{References}

[A] M. Abate: Iteration theory of holomorphic maps on taut manifolds. Mediterranean Press, Cosenza, 1989.

[AP] M. Abate, G. Patrizio: Uniqueness of complex geodesics and characterization of circular domains. Man. Math. 74 (1992), 277-297.

[B] A. Bejancu: Finsler geometry and applications. Ellis Horwood Limited, Chichester, 1990.

[Bu] J. Burbea: On the hessian of the Carathéodory metric. Rocky Mtn. Math. J. 8 (1978), 555-559.

[F] J.J. Faran: Hermitian Finsler metrics and the Kobayashi metric. J. Diff. Geom. 31 (1990), 601-625.

[H] M. Heins: On a class of conformal mappings. Nagoya Math. J. 21 (1962), 1-60.

[K1] S. Kobayashi: Hyperbolic manifolds and holomorphic mappings. Dekker, New York, 1970.

[K2] S. Kobayashi: Negative vector bundles and complex Finsler structures. Nagoya Math. J. 57 (1975), 153-166.

[L] L. Lempert: La métrique de Kobayashi et la représentation des domaines sur la boule. Bull. Soc. Math. France 109 (1981), 427-474.

[P] M.Y. Pang: Finsler metrics with the properties of the Kobayashi metric on convex domains. Preprint, 1990.

[R] H.L. Royden: Complex Finsler metrics. In Contemporary Mathematics. Proceedings of Summer Research Conference, American Mathematical Society, Providence, 1984, pp. 119-124.

[RW] H.L. Royden, P.M. Wong: Carathéodory and Kobayashi metrics on convex domains. Preprint (1983).

[Ru1] H. Rund: The differential geometry of Finsler spaces. Springer, Berlin, 1959.

[Ru2] H. Rund: Generalized metrics on complex manifolds. Math. Nach. 34 (1967), 55-77.

[S] M. Suzuki: The intrinsic metrics on the domains in $\mathbb{C}^{n}$. Math. Rep. Toyama Univ. 6 (1983), 143-177.

[V] E. Vesentini: Complex geodesics. Comp. Math. 44 (1981), 375-394.

[W] B. Wong: On the holomorphic sectional curvature of some intrinsic metrics. Proc. Am. Math. Soc. 65 (1977), 57-61.

[Wu] H. Wu: A remark on holomorphic sectional curvature. Indiana Math. J. 22 (1973), 1103-1108.

Marco Abate

Dipartimento di Matematica

Seconda Università di Roma

00133 Roma, Italy

June 1992
Giorgio Patrizio

Dipartimento di Matematica

Seconda Università di Roma

00133 Roma, Italy 